\title{
THE HARNACK INEQUALITY AND RELATED PROPERTIES FOR SOLUTIONS OF ELLIPTIC AND PARABOLIC EQUATIONS WITH DIVERGENCE-FREE LOWER-ORDER COEFFICIENTS
}

\author{
A. I. NAZAROV AND N. N. URAL'TSEVA
}

To the memory of Mikhail Solomonovich Birman

\begin{abstract}
The paper is devoted to the question as to how "bad" the junior coefficients of elliptic and parabolic equations may be in order that classical properties of their solutions (such as the strict maximum principle, the Harnack inequality and the Liouville theorem) still occur. The answers are given in terms of the Lebesgue and Morrey spaces.
\end{abstract}

\section{§1. INTRODUCTION}

Qualitative properties of solutions of partial differential equations have been studied intensively over the last half of century. In this paper we deal with classical properties, namely, the strong maximum principle, Hölder estimates, the Harnack inequality and the Liouville theorem.

We consider elliptic and parabolic equations of divergence type:

$$
\begin{aligned}
\mathcal{L} u & \equiv-D_{i}\left(a_{i j}(x) D_{j} u\right)+b_{i}(x) D_{i} u=0 ; \\
\mathcal{M} u & \equiv \partial_{t} u-D_{i}\left(a_{i j}(x ; t) D_{j} u\right)+b_{i}(x ; t) D_{i} u=0 .
\end{aligned}
$$

Mostly, we deal with a priori estimates for generalized (sub/super)solutions satisfying the Lipschitz condition. After such estimates are established, we discuss the possibility of their generalization to the case of weak (sub/super)solutions. In this case we assume that $D u \in L_{2, \mathrm{loc}}(\Omega)$ in $(\mathbf{D E})$ and $u \in L_{2, \infty, \mathrm{loc}}(Q), D u \in L_{2, \mathrm{loc}}(Q)$ in $(\mathbf{D P})$.

We always suppose that operators under consideration are uniformly elliptic (parabolic), i.e.,

$$
\nu|\xi|^{2} \leq a_{i j}(\cdot) \xi_{i} \xi_{j} \leq \nu^{-1}|\xi|^{2}, \quad \xi \in \mathbb{R}^{n}
$$

for all values of the arguments, where $\nu$ is a positive constant.

The properties of weak solutions of equations (DE) and (DP) were investigated in a number of papers. Hölder estimates for solutions of (DE) were obtained by De Giorgi [DG] for $\mathbf{b} \equiv 0$ and by Morrey $\left[\mathrm{M}\right.$ ] for $\mathbf{b}$ belonging to the Morrey space lying between $L_{n}$ and any $L_{q}, q>n$ (b stands for $\left(b_{i}\right)$ ). The corresponding result for (DP) was established by Nash [Na] for $\mathbf{b} \equiv 0$ and by Ladyzhenskaya and Ural'tseva [LU1] for $\mathbf{b} \in L_{q+2}, q>n$.

Harnack's inequality for operators without lower-order coefficients was proved by Moser (see [Mo1] for (DE) and [Mo2 for (DP)). Trudinger [Tru proved it for (DE) with $\mathbf{b} \in L_{q}, q>n$. Lieberman (see [Li1, Chapter VI]) extended the result of [Mo2] to

2010 Mathematics Subject Classification. Primary 35B50, 35B53, 35B45.

Key words and phrases. Harnack inequality, Hölder estimates, maximum principle, Liouville theorem.

Partially supported by RFBR (grant no. 08-01-00748) and by grant NSh.4210.2010.1. 
$\mathbf{b} \in L_{q, \ell}, \frac{n}{q}+\frac{2}{\ell}<1$. Obviously, Harnack's inequality implies Hölder estimates. Also, the strong maximum principle follows from Harnack's inequality and the weak maximum principle. Some refinements of the results mentioned, as well as the corresponding results for nondivergence equations, were discussed in our preprint [NU].

In this paper we consider mainly equations (DE) and (DP) with the additional structure condition

$$
\operatorname{div}(\mathbf{b}) \leq 0 \quad \text { in the sense of distributions. }
$$

Equations with lower-order coefficients satisfying this structure condition arise in some applications (see, e.g., [Z, KNSS, CSTY, SSSZ]). We show that in this case the assumptions on $\mathbf{b}$ can be relaxed considerably in the scale of Morrey spaces.

Our paper is organized as follows. In $\S 2$ we deal with elliptic equations. $\S 3$ is devoted to parabolic equations (recall that only the two-sided Liouville theorem holds true for these equations). In $\S 4$ we show an application of our results to some equations arising in hydrodynamics. We emphasize that this section is of illustrative nature, and we do not claim that the results are new.

We recall some notation. $x=\left(x_{1}, \ldots, x_{n}\right)$ is a vector in $\mathbb{R}^{n}, n \geq 2$, with the Euclidean norm $|x| ;(x ; t)$ is a point in $\mathbb{R}^{n+1}$.

$\Omega$ is a domain in $\mathbb{R}^{n}$ and $\partial \Omega$ is its boundary. For a cylinder $\left.Q=\Omega \times\right] 0, T$, we denote by $\left.\partial^{\prime \prime} Q=\partial \Omega \times\right] 0, T\left[\right.$ its lateral surface and by $\partial^{\prime} Q=\partial^{\prime \prime} Q \cup\{\bar{\Omega} \times\{0\}\}$ its parabolic boundary.

We define

$$
\begin{aligned}
B_{R}\left(x^{0}\right) & =\left\{x \in \mathbb{R}^{n}:\left|x-x^{0}\right|<R\right\}, & & B_{R}=B_{R}(0) ; \\
Q_{R}^{\lambda, \theta}\left(x^{0} ; t^{0}\right) & \left.=B_{\lambda R}\left(x^{0}\right) \times\right] t^{0}-\theta R^{2} ; t^{0}[, & & Q_{R}^{\lambda, \theta}=Q_{R}^{\lambda, \theta}(0 ; 0), \quad Q_{R}=Q_{R}^{1,1}
\end{aligned}
$$

(note that $Q_{\lambda R}=Q_{R}^{\lambda, \lambda^{2}}$ ).

The indices $i, j$ vary from 1 to $n$. Repeated indices indicate summation.

The symbol $D_{i}$ denotes the operator of differentiation with respect to $x_{i}$; in particular, $D u=\left(D_{1} u, \ldots, D_{n} u\right)$ is the gradient of $u$. We write $\partial_{t} u$ for the derivative of $u$ with respect to $t$.

The dashed integral denotes the mean value: $f_{E} u=(\text { meas } E)^{-1} \int_{E} u$.

We denote by $\|\cdot\|_{p, \Omega}$ the norm in $L_{p}(\Omega)$. We introduce a scale of anisotropic spaces $L_{q, \ell}(Q)=L_{\ell}(] 0, T\left[\rightarrow L_{q}(\Omega)\right)$ with the norm $\|f\|_{q, \ell, Q}=\|\| f(\cdot ; t)\left\|_{q, \Omega}\right\|_{\ell,] 0, T[}$. Obviously, $L_{q, q}(Q)=L_{q}(Q)$.

We also introduce a scale of Morrey spaces

$$
\mathbb{M}_{q}^{\alpha}(\Omega)=\left\{f \in L_{q}(\Omega):\|f\|_{\mathbb{M}_{q}^{\alpha}(\Omega)} \equiv \sup _{B_{\rho}(x) \subset \Omega} \rho^{-\alpha}\|f\|_{q, B_{\rho}(x)}<\infty\right\} .
$$

The parabolic Morrey spaces $\mathbb{M}_{q, \ell}^{\alpha}(Q)$ are introduced in a similar way, with $Q_{\rho}(x ; t) \subset Q$ instead of $B_{\rho}(x) \subset \Omega$.

Finally, we introduce the space $\mathcal{V}(Q)$ of weak solutions of (DP) with the norm defined by

$$
\|f\|_{\mathcal{V}(Q)}^{2}=\|f\|_{2, \infty, Q}^{2}+\|D f\|_{2,2, Q}^{2} .
$$

We set $f_{+}=\max \{f, 0\}, f_{-}=\max \{-f, 0\}, \operatorname{osc}_{\Omega} f=\sup _{\Omega} f-\inf _{\Omega} f$. For $1 \leq p<n$, $p^{*}=\frac{n p}{n-p}$ is the Sobolev critical exponent.

We use letters $N, C$ (with or without indices) to denote various constants. To indicate that, say, $N$ depends on some parameters, we list them in parentheses: $N(\ldots)$. 


\section{$\S 2$. Elliptid CASE}

Recall that $u$ is a (Lipschitz) subsolution of the equation $\mathcal{L} u=0$ in $\Omega$ (here $\mathcal{L}$ is an operator of the form (DE)) if for any Lipschitz test function $\eta \geq 0$ supported on $\Omega$ we have

$$
\int_{\Omega}\left(a_{i j} D_{j} u D_{i} \eta+b_{i} D_{i} u \eta\right) d x \leq 0 .
$$

We take $\eta=\varphi^{\prime}(u) \cdot \xi$, where $\xi$ is a nonnegative Lipschitz function supported on $B_{\lambda R} \subset \Omega$, while $\varphi \in \mathcal{C}^{2}(\mathbb{R})$ is a convex function vanishing in $\mathbb{R}_{-}$. This gives

$$
\int_{B_{\lambda R} \cap\{u>0\}}\left(a_{i j} D_{j} v D_{i} \xi+\frac{\varphi^{\prime \prime}(u)}{\varphi^{\prime 2}(u)} a_{i j} D_{j} v D_{i} v \xi+b_{i} D_{i} v \xi\right) d x \leq 0,
$$

with $v=\varphi(u)$.

Then, by mollification at a neighborhood of the origin, the assumption $\varphi \in \mathcal{C}^{2}(\mathbb{R})$ can be relaxed to $\varphi \in \mathcal{C}^{2}\left(\mathbb{R}_{+} \cup \mathbb{R}_{-}\right)$.

Lemma 2.1. Let $\mathcal{L}$ be an operator of the form $(\mathbf{D E})$ in $B_{\lambda R}\left(x^{0}\right), \lambda>1$, and let conditions (11) and (2) be satisfied. Also, let $\mathbf{b} \in L_{q}\left(B_{\lambda R}\left(x^{0}\right)\right)$ with some $\frac{n}{2}<q \leq n$.

Then there exists a positive constant $N_{1}$ depending on $n, \nu, \lambda$, q, and the quantity

$$
\mathcal{N}=\mathcal{N}(R, \lambda) \equiv R^{1-\frac{n}{q}}\|\mathbf{b}\|_{q, B_{\lambda R}\left(x^{0}\right)}
$$

such that any Lipschitz subsolution of the equation $\mathcal{L} u=0$ in $B_{\lambda R}\left(x^{0}\right)$ satisfies

$$
\sup _{B_{R}\left(x^{0}\right)} u_{+} \leq N_{1}\left(f_{B_{\lambda R}\left(x^{0}\right)} u_{+}^{2} d x\right)^{\frac{1}{2}}
$$

Proof. We use the classical technique of Moser (see, e.g., [LU2, Chapter IX]). Without loss of generality, we assume that $x^{0}=0$.

In (3), we put $\varphi(\tau)=\tau_{+}^{p}, p>1$, and $\xi=v \zeta^{2}$, where $\zeta$ is a smooth cut-off function in $B_{\lambda R}$. This yields

$$
\int_{B_{\lambda R}}\left(\frac{2 p-1}{p} a_{i j} D_{j} v D_{i} v \zeta^{2}+2 a_{i j} D_{j} v v D_{i} \zeta \zeta+b_{i} D_{i} v v \zeta^{2}\right) d x \leq 0 .
$$

The last term in (5) can be estimated with the help of (2) and the Hölder inequality:

$$
\text { (6) }-\int_{B_{\lambda R}} b_{i} D_{i} v v \zeta^{2} d x \leq \int_{B_{\lambda R}} b_{i} v^{2} \zeta D_{i} \zeta d x \leq\|\mathbf{b}\|_{q, B_{\lambda R}}\|v \zeta\|_{r, B_{\lambda R}}^{2-\frac{1}{s}}\left\|v \zeta^{1-s}|D \zeta|^{s}\right\|_{2, B_{\lambda R}}^{\frac{1}{s}},
$$

where $s>2$ is defined by $\frac{1}{s}=1-\frac{n}{2 q}$, and $r=\frac{2(2 q+n)}{2 q+n-4}$. Note that $2<r<2^{*}$, and, by the embedding theorem,

$$
\begin{aligned}
\|v \zeta\|_{r, B_{\lambda R}} & \leq C(n)(\lambda R)^{n\left(\frac{1}{r}-\frac{1}{2^{*}}\right)}\|D(v \zeta)\|_{2, B_{\lambda R}} \\
& \leq C(n)(\lambda R)^{\frac{1}{2 s-1}}\left(\|D v \zeta\|_{2, B_{\lambda R}}+\|v D \zeta\|_{2, B_{\lambda R}}\right) .
\end{aligned}
$$

Using (11), (6), and (7), from (5) we obtain

$$
\begin{aligned}
\|D v \zeta\|_{2, B_{\lambda R}}^{2} \leq & \frac{1}{\nu} \int_{B_{\lambda R}} a_{i j} D_{j} v D_{i} v \zeta^{2} d x \\
\leq & C_{1}(n, \nu, s, \lambda)\left[\|D v \zeta\|_{2, B_{\lambda R}}\|v D \zeta\|_{2, B_{\lambda R}}\right. \\
& \left.\quad+R^{\frac{1}{s}}\|\mathbf{b}\|_{q, B_{\lambda R}}\left(\|D v \zeta\|_{2, B_{\lambda R}}^{2-\frac{1}{s}}+\|v D \zeta\|_{2, B_{\lambda R}}^{2-\frac{1}{s}}\right)\left\|v \zeta^{1-s}|D \zeta|^{s}\right\|_{2, B_{\lambda R}}^{\frac{1}{s}}\right],
\end{aligned}
$$

\footnotetext{
${ }^{1}$ For $q=n$, the assumption (2) can be lifted. We discuss this at the end of this section.
} 
whence

$$
\|D v \zeta\|_{2, B_{\lambda R}} \leq C_{2}(n, \nu, s, \lambda) \cdot\left[\|v D \zeta\|_{2, B_{\lambda R}}+R\|\mathbf{b}\|_{q, B_{\lambda R}}^{s}\left\|v \zeta^{1-s}|D \zeta|^{s}\right\|_{2, B_{\lambda R}}\right] .
$$

We put $R_{m}=R\left(1+2^{-m}(\lambda-1)\right), m \in \mathbb{N} \cup\{0\}$, and substitute for $\zeta$ the function $\zeta_{m}$ such that

$$
\zeta_{m} \equiv 1 \quad \text { on } \quad B_{R_{m+1}} ; \quad \zeta_{m} \equiv 0 \text { out of } B_{R_{m}} ; \quad \frac{\left|D \zeta_{m}\right|}{\zeta_{m}^{1-\frac{1}{s}}} \leq \frac{2^{m} C_{3}(s)}{(\lambda-1) R} .
$$

Then (8) implies

$$
\left\|D v \zeta_{m}\right\|_{2, B_{R_{m}}} \leq \frac{C_{4}(n, \nu, s, \lambda)}{R} \cdot\|v\|_{2, B_{R_{m}}} \cdot\left(2^{m}+\left(2^{m} \mathcal{N}\right)^{s}\right) .
$$

Now for $p=p_{m} \equiv\left(\frac{r}{2}\right)^{m}$ from (7) and (9) we obtain

$$
\begin{aligned}
\left(f_{B_{R_{m}+1}} u_{+}^{2 p_{m+1}} d x\right)^{\frac{1}{2 p_{m+1}}} & \leq\left(C(n) f_{B_{R_{m}}}\left(v \zeta_{m}\right)^{r} d x\right)^{\frac{1}{r p_{m}}} \\
& \leq\left(2^{2 m s} C_{5} f_{B_{R_{m}}} v^{2} d x\right)^{\frac{1}{2 p_{m}}}=\left(2^{2 m s} C_{5} f_{B_{R_{m}}} u_{+}^{2 p_{m}} d x\right)^{\frac{1}{2 p_{m}}},
\end{aligned}
$$

where $C_{5}$ depends only on $n, \nu, \lambda, s$, and $\mathcal{N}$.

Iterating (10), we arrive at (4).

Corollary 2.1. Suppose $\mathcal{L}$ satisfies the assumptions of Lemma 2.1 in $B_{\lambda R}\left(x^{0}\right)$. If a Lipschitz subsolution of $\mathcal{L} u=0$ in $B_{\lambda R}\left(x^{0}\right)$ satisfies

$$
\operatorname{meas}\left(\{u>k\} \cap B_{\lambda R}\left(x^{0}\right)\right) \leq \mu \operatorname{meas}\left(B_{\lambda R}\right), \quad \mu<N_{1}^{-2},
$$

for some $k$, then

$$
\sup _{B_{R}\left(x^{0}\right)}(u-k) \leq N_{1} \sqrt{\mu} \sup _{B_{\lambda R}\left(x^{0}\right)}(u-k)
$$

(here $N_{1}$ is the constant from Lemma 2.1).

Proof. It suffices to apply Lemma 2.1 to $u-k$.

We need the following version of the embedding theorem.

Proposition A. Let $1 \leq p<n$. Suppose that a nonnegative function $u \in W_{p}^{1}\left(B_{R}\right)$ vanishes on a set $\mathcal{E}_{0}$ of positive measure. Let $\eta=\eta(|x|)$ be a monotone nondecreasing function such that $0 \leq \eta \leq 1$ and $\left.\eta\right|_{\mathcal{E}_{0}} \equiv 1$. Then, for any $1 \leq q \leq p^{*}$ and any measurable set $\mathcal{E} \subset B_{R}$, we have

$$
\|u \eta\|_{q, \mathcal{E}} \leq \frac{C(n) R^{n}}{\operatorname{meas}\left(\mathcal{E}_{0}\right)} \operatorname{meas}^{\frac{1}{q}-\frac{1}{p^{*}}}(\mathcal{E}) \cdot\|D u \eta\|_{p, B_{R}} .
$$

Proof. For $q=p=1$ this proposition was proved in [LSU, Chapter II, Lemma 5.1]. In that lemma, the following inequality was obtained:

$$
\operatorname{meas}\left(\mathcal{E}_{0}\right) \cdot u(x) \eta(x) \leq \frac{(2 R)^{n}}{n} \int_{B_{R}} \frac{|D u(y)| \eta(y)}{|y-x|^{n-1}} d y .
$$

Using the Hardy-Littlewood-Sobolev inequality (see, e.g., [LL, 4.3]), we get

$$
\operatorname{meas}\left(\mathcal{E}_{0}\right) \cdot\|u \eta\|_{p^{*}, B_{R}} \leq C(n, p) R^{n} \cdot\|D u \eta\|_{p, B_{R}},
$$

and the claim follows by the Hölder inequality. 
Lemma 2.2. Suppose $\mathcal{L}$ satisfies the assumptions of Lemma 2.1 in $B_{\lambda R}\left(x^{0}\right)$. Then for any $\delta>0$ there exists a positive constant $\beta$ depending on $n, \nu, \lambda, q, \delta$, and the quantity $\mathcal{N}$ such that if a nonnegative Lipschitz supersolution of the equation $\mathcal{L} V=0$ in $B_{\lambda R}\left(x^{0}\right)$ satisfies

$$
\operatorname{meas}\left(\{V \geq k\} \cap B_{R}\left(x^{0}\right)\right) \geq \delta \cdot \operatorname{meas}\left(B_{R}\right)
$$

for some $k>0$, then

$$
\inf _{B_{R}\left(x^{0}\right)} V \geq \beta k
$$

Proof. Without loss of generality, we may assume $V>0$; otherwise we deal with $V+\varepsilon$ and pass to the limit as $\varepsilon \downarrow 0$. Also, we put $x^{0}=0$.

We define $u=1-\frac{V}{k}$. Note that $u<1$ is a subsolution, so that we can apply relation (3) with $\varphi$ defined only for $\tau<1$.

We put $\varphi(\tau)=\ln _{-}(1-\tau)$ in (3). For $v=\varphi(u)$, this gives

$$
\int_{B_{\lambda R}}\left(a_{i j} D_{j} v D_{i} \xi+a_{i j} D_{j} v D_{i} v \xi+b_{i} D_{i} v \xi\right) d x \leq 0 .
$$

Here we take $\xi=\zeta^{2}$, where $\zeta$ is a smooth cut-off function equal to 1 in $B_{\frac{1+\lambda}{2} R}$. Then, using (11), (2), and the Hölder inequality, we obtain

$$
\begin{aligned}
\|D v \zeta\|_{2, B_{\lambda R}}^{2} & \leq \frac{1}{\nu} \int_{B_{\lambda R}} a_{i j} D_{j} v D_{i} v \zeta^{2} d x \leq \frac{2}{\nu} \int_{B_{\lambda R}}\left(-a_{i j} D_{j} v \zeta D_{i} \zeta+b_{i} v \zeta D_{i} \zeta\right) d x \\
& \leq \frac{2}{\nu}\left(\|D v \zeta\|_{2, B_{\lambda R}}\|D \zeta\|_{2, B_{\lambda R}}+\|\mathbf{b}\|_{q, B_{\lambda R}}\|v \zeta\|_{q^{\prime}, B_{\lambda R}}\|D \zeta\|_{\infty, B_{\lambda R}}\right) .
\end{aligned}
$$

Note that $v$ vanishes on the set $\{V \geq k\} \cap B_{R}$. Therefore, we can apply Proposition A to estimate the last term. By (13), this gives

$$
\|D v \zeta\|_{2, B_{\lambda R}} \leq C_{6}(n, \nu, \lambda, q, \delta) R^{\frac{n}{2}-1} \cdot(1+\mathcal{N}) .
$$

Applying Proposition A once again, we obtain

$$
\left(f_{B_{\frac{1+\lambda}{2} R}} v^{2} d x\right)^{\frac{1}{2}} \leq C_{7},
$$

where $C_{7}$ depends only on $n, \nu, \lambda, q, \delta$. and $\mathcal{N}$.

Finally, relation (15) implies that $v$ is a subsolution. Therefore, we apply Lemma 2.1 to $v$ in $B_{\frac{1+\lambda}{2} R}$ and arrive at the estimate $\sup _{B_{R}} v_{+} \leq C_{8} \equiv N_{1} C_{7}$, which is equivalent to (14) with $\stackrel{2}{\beta}=\exp \left(-C_{8}\right)$.

Corollary 2.2 (strong maximum principle). Let $\mathcal{L}$ be an operator of the form $(\mathbf{D E})$ in $\Omega$, and let conditions (11) and (2) be satisfied. Also, let $\mathbf{b} \in L_{q, \operatorname{loc}}(\Omega)$ with some $\frac{n}{2}<q \leq n$. Then no nonconstant Lipschitz supersolution of the equation $\mathcal{L} V=0$ in $\Omega$ can attain its minimum at an interior point of $\Omega$.

Proof. Suppose the contrary. Without loss of generality, $\inf _{\Omega} V=0$. Then there exists $x^{0} \in \Omega$ that is a boundary point of the set $\{V>0\}$. Choose $R$ such that $\overline{B_{2 R}}\left(x^{0}\right) \subset \Omega$. Then relation (13) is true for some $k>0$ and $\delta>0$, and we obtain (14), a contradiction.

Lemma 2.3. Suppose $\mathcal{L}$ satisfies the assumptions of Lemma 2.1 in $B_{3 R}$. Then any Lipschitz solution of the equation $\mathcal{L} u=0$ in $B_{3 R}$ satisfies the estimate

$$
\underset{B_{R}}{\operatorname{OSc}} u \leq \varkappa_{0} \underset{B_{3 R}}{\operatorname{OSc}} u
$$

where $\varkappa_{0}<1$ depends on $n, \nu, q$, and the quantity $\mathcal{N}$. 
Proof. We set

$$
k=\frac{1}{2}\left(\sup _{B_{3 R}} u+\inf _{B_{3 R}} u\right)
$$

and consider two cases.

1. Suppose relation (11) is fulfilled with $\lambda=2$ and $\mu=\frac{1}{4} N_{1}^{-2}$. Then, by Corollary 2.1,

$$
\sup _{B_{R}} u \leq \frac{1}{2}\left(\sup _{B_{2 R}} u+k\right) \leq \sup _{B_{3 R}} u-\frac{1}{4} \underset{B_{3 R}}{\operatorname{osc}} u .
$$

2. Otherwise, we apply Lemma 2.2 (with $\lambda=\frac{3}{2}$ and $\delta=\mu$ ) to the (nonnegative) function $V=u-\inf _{B_{3 R}} u$. This gives $\inf _{B_{R}} V \geq \inf _{B_{2 R}} V \geq \beta\left(k-\inf _{B_{3 R}} u\right)$, whence

$$
\inf _{B_{R}} u \geq \inf _{B_{3 R}} u+\frac{\beta}{2} \underset{B_{3 R}}{\operatorname{OSc}} u .
$$

In both cases we arrive at (16) with $\varkappa_{0}=\min \left\{\frac{1}{4}, \frac{\beta}{2}\right\}$.

Corollary 2.3 (Hölder estimate). Suppose $\mathcal{L}$ satisfies the assumptions of Lemma 2.1 in $B_{R_{0}}$. Also, let $\sup _{R<R_{0}} \mathcal{N}(R, 1)<\infty$.

Then any Lipschitz solution of the equation $\mathcal{L} u=0$ in $B_{R_{0}}$ satisfies the estimate

$$
\underset{B_{\rho}}{\operatorname{osc}} u \leq N_{2}\left(\frac{\rho}{r}\right)^{\gamma} \cdot \underset{B_{r}}{\operatorname{osc}} u, \quad 0<\rho<r \leq R_{0},
$$

where $N_{2}$ and $\gamma$ depend on $n, \nu, q$, and $\sup _{R<R_{0}} \mathcal{N}(R, 1)$.

Proof. Iterating estimate (16), we arrive at (17) with $\gamma=-\log _{3}\left(\varkappa_{0}\right)$.

Corollary 2.4 (two-sided Liouville theorem). Let $\mathcal{L}$ be an operator of the form (DE) in $\mathbb{R}^{n}$, and let conditions (11) and (2) be satisfied. Also, let $\mathbf{b} \in L_{q, \text { loc }}\left(\mathbb{R}^{n}\right)$ with some $\frac{n}{2}<q \leq n$. Finally, assume that

$$
\liminf _{R \rightarrow \infty} \mathcal{N}(R, 1)<\infty .
$$

Then any bounded Lipschitz solution of the equation $\mathcal{L} u=0$ in $\mathbb{R}^{n}$ is constant.

Remark 1. If $\mathbf{b} \in L_{q}\left(\mathbb{R}^{n}\right)$, then, obviously, (18) is satisfied.

Proof. Iteration of (16) with respect to a suitable sequence $R_{m} \rightarrow \infty$ gives the claim.

Lemma 2.4. Let $\mathcal{L}$ be an operator of the form (DE) in $B_{2 R}$, and let conditions (1) and (2) be satisfied. Also, let $\mathbf{b} \in \mathbb{M}_{q}^{\frac{n}{q}-1}\left(B_{2 R}\right)$ with some $\frac{n}{2}<q \leq n$.

Suppose that, for a nonnegative Lipschitz supersolution of the equation $\mathcal{L} V=0$ in $B_{2 R}$ and for some $y \in B_{2 R}$, the inequality $\inf _{B_{\rho}(y)} V=k>0$ is valid with $\rho=\frac{1}{4} \operatorname{dist}\left(y, \partial B_{2 R}\right)$. Then

$$
\inf _{B_{R}} V \geq \widehat{\beta}\left(\frac{\rho}{R}\right)^{\widehat{\gamma}} k
$$

where $\widehat{\beta}$ and $\widehat{\gamma}$ depend on $n, \nu, q$, and $\|\mathbf{b}\|_{\mathbb{M}_{q}^{\frac{n}{q}-1}\left(B_{2 R}\right)}$.

Proof. Denote by $\mathfrak{N}$ an integer such that $2^{-(\mathfrak{N}+1)} R<\rho \leq 2^{-\mathfrak{N}} R$ and consider the ball $B_{\mathfrak{r}_{0}}\left(y^{0}\right)$, where $\mathfrak{r}_{0}=2^{-\mathfrak{N}} R, y^{0}=2 R\left(1-2^{-\mathfrak{N}}\right) \mathbf{e}$, and $\mathbf{e}=\frac{y}{|y|}$. It is easily seen that $B_{\mathfrak{r}_{0}}\left(y^{0}\right) \subset B_{3 \rho}(y)$, and by Lemma 2.2 (with $\lambda=\frac{4}{3}$ and $\delta=\frac{1}{3^{n}}$ ), we have

$$
\inf _{B_{\mathfrak{r}_{0}}\left(y^{0}\right)} V \geq \inf _{B_{3 \rho}(y)} V \geq \beta k .
$$

Now we introduce a sequence of balls $B_{\mathfrak{r}_{m}}\left(y^{m}\right), m=1, \ldots, \mathfrak{N}$, as follows:

$$
\mathfrak{r}_{m}=2 \mathfrak{r}_{m-1}, \quad y^{m}=y^{m-1}-\mathfrak{r}_{m} \mathbf{e} .
$$


For all $m=1, \ldots, \mathfrak{N}$ we have

$$
B_{2 \mathfrak{r}_{m}}\left(y^{m}\right) \subset B_{2 R} ; \quad \operatorname{meas}\left(B_{\mathfrak{r}_{m-1}}\left(y^{m-1}\right) \cap B_{\mathfrak{r}_{m}}\left(y^{m}\right)\right) \geq C(n) \cdot \operatorname{meas}\left(B_{\mathfrak{r}_{m}}\right) .
$$

Thus, Lemma 2.2 (with $\lambda=2$ and $\delta=C(n)$ ) gives

$$
\inf _{B_{\mathbf{r}_{m}}\left(y^{m}\right)} V \geq \beta \cdot \inf _{B_{\mathfrak{r}_{m-1}}\left(y^{m-1}\right)} V .
$$

Since $B_{\mathfrak{r}_{\mathfrak{N}}}\left(y^{\mathfrak{N}}\right)=B_{R}$, we obtain

$$
\inf _{B_{R}} V \geq \beta^{\mathfrak{N}} \cdot \inf _{B_{\mathfrak{r}_{0}}\left(y^{0}\right)} V \geq\left(\frac{\rho}{R}\right)^{\widehat{\gamma}} \cdot \inf _{B_{\mathfrak{r}_{0}}\left(y^{0}\right)} V,
$$

where $\widehat{\gamma}=-\log _{2}(\beta)$.

Combining this with (20), we arrive at (19).

Theorem 2.5 (the Harnack inequality). Suppose that $\mathcal{L}$ satisfies the assumptions of Lemma 2.4 in $B_{2 R}$. Then there exists a positive constant $N_{3}$ depending on $n, \nu, q$, and $\|\mathbf{b}\|_{\mathbb{M}_{q}^{\frac{n}{q}-1}\left(B_{2 R}\right)}$ such that any nonnegative Lipschitz solution of the equation $\mathcal{L} u=0$ in $B_{2 R}$ satisfies

$$
\sup _{B_{R}} u \leq N_{3} \cdot \inf _{B_{R}} u
$$

Proof. We follow the idea of Safonov (see [S2]). Denote by $y \in B_{2 R}$ a maximum point of the function

$$
v(x)=\left(\operatorname{dist}\left(x, \partial B_{2 R}\right)\right)^{\widehat{\gamma}} \cdot u(x)
$$

(here $\widehat{\gamma}$ is the constant from Lemma 2.4) and set

$$
\rho=\frac{1}{4} \operatorname{dist}\left(y, \partial B_{2 R}\right), \quad \mathfrak{M}=v(y)=(4 \rho)^{\widehat{\gamma}} \cdot u(y) .
$$

Obviously,

$$
\begin{aligned}
\sup _{B_{R}} u & \leq \frac{\mathfrak{M}}{R^{\widehat{\gamma}}}=\left(\frac{4 \rho}{R}\right)^{\widehat{\gamma}} \cdot u(y), \\
\sup _{B_{2 \rho}(y)} u & \leq \frac{\mathfrak{M}}{(2 \rho)^{\widehat{\gamma}}}=2^{\widehat{\gamma}} \cdot u(y) .
\end{aligned}
$$

Denote $k=\frac{1}{2} u(y)$. If meas $\left(\{u>k\} \cap B_{2 \rho}(y)\right) \leq \mu$ meas $\left(B_{2 \rho}\right)$, then Corollary 2.1 (with $\lambda=2$ ) and (23) imply the relation

$$
k=u(y)-k \leq \sup _{B_{\rho}(y)}(u-k) \leq N_{1} \sqrt{\mu} \sup _{B_{2 \rho}(y)}(u-k) \leq N_{1} \sqrt{\mu}\left(2^{\widehat{\gamma}+1}-1\right) k,
$$

which is impossible for $\mu \leq \mu_{0} \equiv \frac{1}{2^{2 \hat{\gamma}+2}} N_{1}^{-2}$. Thus, meas $\left(\{u>k\} \cap B_{2 \rho}(y)\right) \geq$ $\mu_{0}$ meas $\left(B_{2 \rho}\right)$, and Lemma 2.2 (with $\lambda=2$ and $\left.\delta=\mu_{0}\right)$ gives

$$
\inf _{B_{\rho}(y)} u \geq \inf _{B_{2 \rho}(y)} u \geq \beta k=\frac{\beta}{2} \cdot u(y) .
$$

Finally, Lemma 2.4 shows that

$$
\inf _{B_{R}} u \geq \widehat{\beta}\left(\frac{\rho}{R}\right)^{\widehat{\gamma}} \inf _{B_{\rho}(y)} u .
$$

Combining (22), (24), and (25), we arrive at (21) with $N_{3}=\frac{2^{2 \hat{\gamma}+1}}{\beta \widehat{\beta}}$. 
Theorem 2.6 (one-sided Liouville theorem). Let $\mathcal{L}$ be an operator of the form (DE) in $\mathbb{R}^{n}$, and let conditions (11) and (2) be satisfied. Also, let $\mathbf{b} \in \mathbb{M}_{q, \text { loc }}^{\frac{n}{q}-1}\left(\mathbb{R}^{n}\right)$ with some $\frac{n}{2}<q \leq n$, and assume that, for some $\delta>0$, we have

$$
\liminf _{R \rightarrow \infty} \sup _{|x|=R}\|\mathbf{b}\|_{\mathbb{M}_{q}^{\frac{n}{q}-1}\left(B_{\delta R}(x)\right)}<\infty .
$$

Then any semibounded Lipschitz solution of the equation $\mathcal{L} u=0$ in $\mathbb{R}^{n}$ is constant.

Remark 2. If $\mathbf{b} \in \mathbb{M}_{q}^{\frac{n}{q}-1}\left(\mathbb{R}^{n}\right)$, then, obviously, (26) is satisfied.

Proof. Without loss of generality, we may assume that $u$ is bounded from below, and that $\inf _{\mathbb{R}^{n}} u=0$.

We take a sequence $R_{m} \rightarrow \infty$ such that $\mathfrak{B} \equiv \sup _{m} \sup _{|x|=R_{m}}\|\mathbf{b}\|_{\mathbb{M}_{q}^{\frac{n}{q}-1}\left(B_{\delta R}(x)\right)}<\infty$. Next, we cover the sphere $|x|=1$ with a finite set of balls $B_{\frac{\delta}{2}}(x)$ and dilate these balls to obtain a covering of the sphere $|x|=R_{m}$. Applying Theorem 2.5 to all the balls of this covering, we see that $\sup _{|x|=R_{m}} u \leq C(n, \nu, q, \mathfrak{B}, \delta) \cdot \inf _{|x|=R_{m}} u$ for any $m$. By Corollary 2.2,

$$
\sup _{B_{R}} u=\sup _{|x|=R} u ; \quad \inf _{|x|=R} u=\inf _{B_{R}} u \rightarrow 0 \quad \text { as } \quad R \rightarrow \infty,
$$

and the claim follows.

Now, we briefly discuss the possibility to generalize all previous statements to the case of weak (sub/super)solutions.

The proof of Lemma 2.1 runs without changes 2 also for weak subsolutions of the equation $\mathcal{L} u=0$ if the bilinear form

$$
\mathcal{B}\langle u, \eta\rangle \equiv \int_{B_{\lambda R}} b_{i} D_{i} u \eta d x
$$

extends by continuity to the pairs $\left(v, v \zeta^{2}\right)$ with $D v \in L_{2}\left(B_{\lambda R}\right)$. This is certainly true provided

$$
|\mathcal{B}\langle u, \eta\rangle| \leq C\|D u\|_{2, B_{2 \lambda R}}\|D \eta\|_{2, B_{2 \lambda R}}, \quad u, \eta \in \mathcal{C}_{0}^{\infty}\left(B_{2 \lambda R}\right) .
$$

In MV] it was shown that the last estimate is valid if

$$
\Delta^{-1} \operatorname{curl}(\mathbf{b}) \in B M O^{n \times n}\left(B_{2 \lambda R}\right) ; \quad h=\left|\nabla\left(\Delta^{-1} \operatorname{div}(\mathbf{b})\right)\right|^{2} \in \mathfrak{M}_{+}^{1,2}
$$

(here we assume that $\mathbf{b}$ is extended by zero); the last notation means the class of the so-called admissible weights, i.e., those satisfying

$$
\int_{B_{2 \lambda R}} h|v|^{2} d x \leq C\|D v\|_{2, B_{2 \lambda R}}^{2}, \quad v \in \mathcal{C}_{0}^{\infty}\left(B_{2 \lambda R}\right) .
$$

If $\mathbf{b} \in \mathbb{M}_{q}^{\frac{n}{q}-1}\left(B_{\lambda R}\right)$, the first relation in (27) follows from elliptic coercive estimates and the Poincaré inequality, see, e.g., Tro. Thus, Lemma 2.1 and, with it, all subsequent statements hold true for weak (sub/super)solutions of the equation $\mathcal{L} u=0$ if, for example, $\mathbf{b} \in \mathbb{M}_{q}^{\frac{n}{q}-1}$ and $\operatorname{div}(\mathbf{b}) \equiv 0$.

Separately, we consider the case where $q=n$. Now the space $\mathbb{M}_{q}^{\frac{n}{q}-1}(\Omega)$ becomes the conventional Lebesgue space $L_{n}(\Omega)$, and we claim that the main results of this section

\footnotetext{
${ }^{2}$ More formally, in this case inequality (3) is fulfilled under the additional condition that $\varphi$ is globally Lipschitz. Thus, to derive (8) one should take $\varphi^{\prime}(u)=p \min \left\{u_{+}, N\right\}^{p-1}, N>0, \xi=\varphi(u) \zeta^{2}$, and then pass to the limit as $N \rightarrow \infty$.
} 
hold true without the assumption (2) 3 . Note that in this case relation (5) is fulfilled for any weak subsolution $u$.

First, let $n \geq 3$. Then, estimating the last term in (5) by the Hölder inequality and the Sobolev inequality, we obtain an analog of (8):

$$
\|D v \zeta\|_{2, B_{\lambda R}} \leq C_{2}^{\prime}(n, \nu) \cdot\left[\|v D \zeta\|_{2, B_{\lambda R}}+\|\mathbf{b}\|_{n, B_{\lambda R}}\|D v \zeta\|_{2, B_{\lambda R}}\right] .
$$

If $\|\mathbf{b}\|_{n, B_{\lambda R}} \leq \varepsilon(n, \nu) \equiv\left(2 C_{2}^{\prime}\right)^{-1}$, then for $p=p_{m} \equiv 2\left(\frac{n}{n-2}\right)^{m}$ we obtain an analog of (10):

$$
\left(f_{B_{R_{m}+1}} u_{+}^{2 p_{m+1}} d x\right)^{\frac{1}{2 p_{m+1}}} \leq\left(C_{5}^{\prime} f_{B_{R_{m}}} u_{+}^{2 p_{m}} d x\right)^{\frac{1}{2 p_{m}}}
$$

where $C_{5}^{\prime}$ depends only on $n, \nu$, and $\lambda$. The remaining part of the proof of Lemma 2.1 runs without changes.

In a similar way, we can prove Lemmas 2.2 and 2.4 for weak supersolutions of the equation $\mathcal{L} V=0$ under the same assumption $\|\mathbf{b}\|_{n, B_{\lambda R}} \leq \varepsilon(n, \nu)$ (in Lemma 2.4 we put $\lambda=2)$.

If $n=2$, we use the Yudovich-Pohozhaev embedding theorem (see, e.g., [BIN, 10.6]) instead of the Sobolev inequality. This gives us Lemmas 2.1, 2.2, 2.4 under the assumption $\left\|\mathbf{b} \ln ^{\frac{1}{2}}(1+\lambda R|\mathbf{b}|)\right\|_{2, B_{\lambda R}\left(x^{0}\right)} \leq \varepsilon(n, \nu)$.

Next, the strong maximum principle holds true without any smallness assumptions on $\mathbf{b}$. Indeed, one can choose $R$ so small that these assumptions are fulfilled.

Since the proof of Theorem 2.5 depends only on Lemmas 2.1, 2.2, 2.4, the Harnack inequality is obviously valid under the smallness assumption on b. However, we can eliminate this assumption by using a trick of M. V. Safonov, see [S2].

Theorem 2.5' (the Harnack inequality). Let $\mathcal{L}$ be an operator of the form $(\mathbf{D E})$ in $B_{2 R}$, and let condition (11) be satisfied. Also, suppose that

$$
\|\mathbf{b}\|_{n, B_{2 R}} \leq \mathfrak{B} \quad \text { for } \quad n \geq 3 ; \quad\left\|\mathbf{b} \ln ^{\frac{1}{2}}(1+R|\mathbf{b}|)\right\|_{2, B_{2 R}} \leq \mathfrak{B} \quad \text { for } \quad n=2 .
$$

Then there exists a positive constant $N_{3}^{\prime}$ depending only on $n, \nu$, and $\mathfrak{B}$ such that any nonnegative weak solution of the equation $\mathcal{L} u=0$ in $B_{2 R}$ satisfies

$$
\sup _{B_{R}} u \leq N_{3}^{\prime} \cdot \inf _{B_{R}} u \text {. }
$$

Proof. We split the spherical layer $B_{2 R} \backslash B_{R}$ into $M$ layers of equal thickness $\frac{R}{M}$ and put $\delta=\frac{1}{2 M}$. Obviously, we can choose $M$ depending only on $n, \nu$, and $\mathfrak{B}$ and such that for at least one of these layers (say, $K=\{r-2 \delta R<|x|<r+2 \delta R\}$ ) the following estimates are true:

$$
\|\mathbf{b}\|_{n, K} \leq \varepsilon \quad \text { for } \quad n \geq 3 ; \quad\left\|\mathbf{b} \ln ^{\frac{1}{2}}(1+2 \delta R|\mathbf{b}|)\right\|_{2, K} \leq \varepsilon \quad \text { for } \quad n=2
$$

(here $\varepsilon=\varepsilon(n, \nu)$ is the smallness constant introduced above).

We cover the sphere $|x|=r$ with a finite set of balls $B_{\delta R}(x)$ (note that the number of balls depends only on $\delta$ ). Since all doubled balls $B_{2 \delta R}(x)$ lye in $K$, we can apply Harnack's inequality in these balls. This gives $\sup _{|x|=r} u \leq C(n, \nu, \delta) \cdot \inf |x|=r u$. However, by the maximum principle,

$$
\inf _{B_{R}} u \geq \inf _{|x|=r} u, \quad \sup _{B_{R}} u \leq \sup _{|x|=r} u
$$

and the claim follows.

\footnotetext{
${ }^{3}$ Moreover, in this case the assumption $\mathbf{b} \in L_{n}$ can be relaxed in the scale of Lorentz spaces to $\mathbf{b} \in \Lambda_{n, q}$ with any $q<\infty$. We do not discuss this here for the lack of place.
} 
The following statement can be proved by a word-for-word repetition of the proof of Theorem 2.6, with the use of Theorem $2.5^{\prime}$.

Theorem 2.6' (the Liouville theorem). Let $\mathcal{L}$ be an operator of the form $(\mathbf{D E})$ in $\mathbb{R}^{n}$. Let condition (1) be satisfied, and let

$$
\mathbf{b} \in L_{n, \text { loc }}\left(\mathbb{R}^{n}\right) \quad \text { for } \quad n \geq 3 ; \quad \mathbf{b} \ln ^{\frac{1}{2}}(1+|\mathbf{b}|) \in L_{2, \text { loc }}\left(\mathbb{R}^{2}\right) \quad \text { for } \quad n=2 .
$$

Also, suppose that for some $\delta>0$ we have

$$
\begin{aligned}
& \liminf _{R \rightarrow \infty} \sup _{|x|=R}\|\mathbf{b}\|_{n, B_{\delta R}(x)}<\infty, \quad n \geq 3 ; \\
& \liminf _{R \rightarrow \infty} \sup _{|x|=R}\left\|\mathbf{b} \ln ^{\frac{1}{2}}(1+R|\mathbf{b}|)\right\|_{2, B_{\delta R}(x)}<\infty, \quad n=2 .
\end{aligned}
$$

Then any weak semibounded solution of the equation $\mathcal{L} u=0$ in $\mathbb{R}^{n}$ is constant.

Remark 3. If $\mathbf{b} \in L_{n}\left(\mathbb{R}^{n}\right)$ (respectively, $\mathbf{b} \ln ^{\frac{1}{2}}(1+|x||\mathbf{b}|) \in L_{2}\left(\mathbb{R}^{2}\right)$ ), then, obviously, (30) is satisfied.

As to Hölder estimates for solutions, we have two possibilities. The first is to take, in the proof of Lemma 2.3, $R$ to be so small that the smallness assumptions on $\mathbf{b}$ are satisfied in $B_{2 R}$. This gives estimate (17) with $\gamma$ depending only on $n$ and $\nu$, while $N_{2}$ depends also on the modulus of continuity of $\mathbf{b}$ in $L_{n}\left(B_{R_{0}}\right)$ (respectively, of $\mathbf{b} \ln ^{\frac{1}{2}}(1+R|\mathbf{b}|)$ in $\left.L_{2}\left(B_{R_{0}}\right)\right)$. The second possibility is to use Theorem $2.5^{\prime}$. This gives (17) with both $\gamma$ and $N_{2}$ depending on $n, \nu$, and $\|\mathbf{b}\|_{n, B_{R_{0}}}$ (respectively, $\left\|\mathbf{b} \ln ^{\frac{1}{2}}\left(1+R_{0}|\mathbf{b}|\right)\right\|_{2, B_{R_{0}}}$ ).

\section{$\S 3$. Parabolic case}

Lemma 3.1. Let $\mathcal{M}$ be an operator of the form (DP) in $Q_{R}^{\lambda, \theta}\left(x^{0} ; t^{0}\right)$ with $\lambda>1, \theta>0$, and let conditions (11) and (2) be satisfied. Also, let $\mathbf{b} \in L_{q, \ell}\left(Q_{R}^{\lambda, \theta}\left(x^{0} ; t^{0}\right)\right)$ with some $q$ and $\ell$ such that

$$
\alpha=\alpha(q, \ell) \equiv \frac{n}{q}+\frac{2}{\ell}-1 \in[0,1[
$$

( $q$ and/or $\ell$ may be infinit $\left.\S^{4}\right)$.

Then there exists a positive constant $N_{4}$ depending on $n, \nu, \lambda, \theta, q, \ell$, and the quantity

$$
\widehat{\mathcal{N}}=\widehat{\mathcal{N}}(R, \lambda, \theta) \equiv R^{-\alpha}\|\mathbf{b}\|_{q, \ell, Q_{R}^{\lambda, \theta}\left(x^{0} ; t^{0}\right)}
$$

such that any Lipschitz subsolution of the equation $\mathcal{M} u=0$ in $Q_{R}^{\lambda, \theta}\left(x^{0} ; t^{0}\right)$ satisfies

$$
\sup _{Q_{R}^{1, \frac{\theta}{2}}\left(x^{0} ; t^{0}\right)} u_{+} \leq N_{4}\left(f_{Q_{R}^{\lambda, \theta}\left(x^{0} ; t^{0}\right)} u_{+}^{2} d x d t\right)^{\frac{1}{2}} .
$$

Remark 4 . The quantity $\widehat{\mathcal{N}}$ depends also on $q$ and $\ell$. However, we regard these parameters as fixed, and we do not indicate this dependence. We also do not indicate the dependence of $\widehat{\mathcal{N}}$ on $x^{0}$ and $t^{0}$.

Proof. We argue much as in Lemma 2.1. There is no loss of generality in assuming that $\left(x^{0} ; t^{0}\right)=(0 ; 0)$.

For a nonnegative test function $\eta$ we have

$$
\int_{Q_{R}^{\lambda, \theta}}\left(\partial_{t} u \eta+a_{i j} D_{j} u D_{i} \eta+b_{i} D_{i} u \eta\right) d x d t \leq 0 .
$$

\footnotetext{
${ }^{4}$ For $\alpha=0$, the assumption (2) can be lifted, with some reservations in the case where $q=n, \ell=\infty$. We discuss this at the end of this section.
} 
We take $\eta=\varphi^{\prime}(u) \cdot \xi$, where $\xi$ is a cut-off function that is Lipschitz in $x$ and vanishes on a neighborhood of $\partial^{\prime} Q_{R}^{\lambda, \theta}$, while $\varphi \in \mathcal{C}^{2}(\mathbb{R})$ is a convex function vanishing in $\mathbb{R}_{-}$. This gives

$$
\int_{Q_{R}^{\lambda, \theta} \cap\{u>0\}}\left(\partial_{t} v \xi+a_{i j} D_{j} v D_{i} \xi+\frac{\varphi^{\prime \prime}(u)}{\varphi^{\prime 2}(u)} a_{i j} D_{j} v D_{i} v \xi+b_{i} D_{i} v \xi\right) d x d t \leq 0
$$

where $v=\varphi(u)$.

As in $\S 2$, by mollification in a neighborhood of the origin, in (3) the assumption $\varphi \in \mathcal{C}^{2}(\mathbb{R})$ can be relaxed to $\varphi \in \mathcal{C}^{2}\left(\mathbb{R}_{+} \cup \mathbb{R}_{-}\right)$.

Now in (33) we put $\varphi(\tau)=\tau_{+}^{p}, p>1$, and $\xi=\chi_{\{t<\bar{t}\}} \cdot v \zeta^{2}$, where $\zeta$ is a smooth cut-off function in $\left.Q_{R}^{\lambda, \theta}, \bar{t} \in\right]-\theta R^{2}, 0[$. Then

$$
\begin{array}{r}
\left.\frac{1}{2} \int_{B_{\lambda R}}(v \zeta)^{2}\right|^{t=\bar{t}} d x+\int_{Q_{R}^{\lambda, \theta}} \chi_{\{t<\bar{t}\}}\left(\frac{2 p-1}{p} a_{i j} D_{j} v D_{i} v \zeta^{2}+2 a_{i j} D_{j} v v D_{i} \zeta \zeta\right. \\
\left.-v^{2} \zeta \partial_{t} \zeta+b_{i} D_{i} v v \zeta^{2}\right) d x d t \leq 0 .
\end{array}
$$

The last term in (34) can be estimated by using (2) and the Hölder inequality:

$$
\begin{aligned}
-\int_{Q_{R}^{\lambda, \theta}} \chi_{\{t<\bar{t}\}} b_{i} D_{i} v v \zeta^{2} d x d t & \leq \int_{Q_{R}^{\lambda, \theta}} \chi_{\{t<\bar{t}\}} b_{i} v^{2} \zeta D_{i} \zeta d x d t \\
& \leq\|\mathbf{b}\|_{q, \ell, Q_{R}^{\lambda, \theta}}\|v \zeta\|_{r, l, Q_{R}^{\lambda, \theta}}^{2-\frac{1}{s}}\left\|v \zeta^{1-s}|D \zeta|^{s}\right\|_{2,2, Q_{R}^{\lambda, \theta}}^{\frac{1}{s}},
\end{aligned}
$$

where $s>2$ is defined by $\frac{1}{s}=1-\frac{n}{2 q}-\frac{1}{\ell}$, while $r$ and $l$ are defined by

$$
\frac{1}{2 s}+\frac{1}{q}+\frac{2-\frac{1}{s}}{r}=1 ; \quad \frac{1}{2 s}+\frac{1}{\ell}+\frac{2-\frac{1}{s}}{l}=1 .
$$

Note that $\frac{n}{2}<\frac{n}{r}+\frac{2}{l}<\frac{n}{2}+1$, and, by the embedding theorem [LSU, Chapter II, (3.4)],

$$
\|v \zeta\|_{r, l, Q_{R}^{\lambda, \theta}} \leq C_{9}(n, r, l, \lambda, \theta) R^{\frac{n}{r}+\frac{2}{l}-\frac{n}{2}}\|v \zeta\|_{\mathcal{V}\left(Q_{R}^{\lambda, \theta}\right)} .
$$

Using (11), (35), (36), and the Young inequality, from (34) we obtain

$$
\begin{aligned}
\|v \zeta\|_{\mathcal{V}\left(Q_{R}^{\lambda, \theta}\right)}^{2} \leq & C_{10}(n, \nu, q, \ell, \lambda, \theta) \\
& \times \int_{Q_{R}^{\lambda, \theta}} v^{2}\left(|D \zeta|^{2}+\zeta\left|\partial_{t} \zeta\right|+R^{2}\|\mathbf{b}\|_{q, \ell, Q_{R}^{\lambda, \theta}}^{2 s} \zeta^{2-2 s}|D \zeta|^{2 s}\right) d x d t .
\end{aligned}
$$

We put $\lambda_{m}=1+2^{-m}(\lambda-1), \theta_{m}=\frac{\theta}{2}\left(1+4^{-m}\right), m \in \mathbb{N} \cup\{0\}$, and substitute $\zeta=\zeta_{m}$ such that

$$
\begin{gathered}
\zeta_{m} \equiv 1 \quad \text { in } Q_{R}^{\lambda_{m+1}, \theta_{m+1}} ; \quad \zeta_{m} \equiv 0 \text { outside of } Q_{R}^{\lambda_{m}, \theta_{m}} \\
\left|\partial_{t} \zeta_{m}\right| \leq \frac{4^{m} C}{\theta R^{2}} ; \quad \frac{\left|D \zeta_{m}\right|}{\zeta_{m}^{1-\frac{1}{s}}} \leq \frac{2^{m} C_{3}(s)}{(\lambda-1) R}
\end{gathered}
$$

Then (37) implies

$$
\left\|v \zeta_{m}\right\|_{\mathcal{V}\left(Q_{R}^{\lambda_{m}, \theta_{m}}\right)} \leq \frac{C_{11}(n, \nu, q, \ell, \lambda, \theta)}{R} \cdot\|v\|_{2,2, Q_{R}^{\lambda_{m}, \theta_{m}}} \cdot\left(2^{m}+\left(2^{m} \widehat{\mathcal{N}}\right)^{s}\right) .
$$

Now, for $p=p_{m} \equiv\left(\frac{n+2}{n}\right)^{m}$, from (36) (with $r=l=\frac{2(n+2)}{n}$ ) and (38) we deduce that

$$
\begin{gathered}
\left(f_{Q_{R}^{\lambda_{m}+1, \theta_{m+1}}} u_{+}^{2 p_{m+1}} d x d t\right)^{\frac{1}{2 p_{m}+1}} \leq\left(C(n) f_{Q_{R}^{\lambda_{m}, \theta_{m}}}\left(v \zeta_{m}\right)^{r} d x d t\right)^{\frac{1}{r p_{m}}} \\
\leq\left(2^{2 m s} C_{12} f_{Q_{R}^{\lambda_{m}, \theta_{m}}} v^{2} d x d t\right)^{\frac{1}{2 p_{m}}}=\left(2^{2 m s} C_{12} f_{Q_{R}^{\lambda_{m}, \theta_{m}}} u_{+}^{2 p_{m}} d x d t\right)^{\frac{1}{2 p_{m}}},
\end{gathered}
$$


where $C_{12}$ depends only on $n, \nu, q, \ell, \lambda, \theta$, and $\widehat{\mathcal{N}}$.

Iterating (39) we arrive at (32).

Remark 5. If $\mathbf{b}=\mathbf{b}^{(1)}+\mathbf{b}^{(2)}$, and $\mathbf{b}^{(j)} \in L_{q_{j}, \ell_{j}}\left(Q_{R}^{\lambda, \theta}\left(x^{0} ; t^{0}\right)\right), j=1,2$, with $\alpha\left(q_{j}, \ell_{j}\right) \in$ $[0,1[$, then the proof of Lemma 3.1 does not change. The same is true for the other statements of this section.

Remark 6. Under the assumptions of Lemma 3.1, if $u$ satisfies

$$
u\left(\cdot ; t^{0}-\theta R^{2}\right) \leq 0 \quad \text { in } \quad B_{\lambda R}\left(x^{0}\right),
$$

then we can estimate $u$ up to the bottom of the cylinder, i.e., the left-hand side in (32) can be replaced by $\sup _{Q_{R}^{1, \theta}\left(x^{0} ; t^{0}\right)} u_{+}$. Indeed, we can simply put $\theta_{m} \equiv \theta$ and take $\zeta_{m}$ independent on $t$.

Corollary 3.1. Suppose $\mathcal{M}$ satisfies the assumptions of Lemma 3.1 in $Q_{R}^{\lambda, \theta}\left(x^{0} ; t^{0}\right)$.

1. If a Lipschitz subsolution of $\mathcal{M} u=0$ in $Q_{R}^{\lambda, \theta}\left(x^{0} ; t^{0}\right)$ satisfies

$$
\operatorname{meas}\left(\{u>k\} \cap Q_{R}^{\lambda, \theta}\left(x^{0} ; t^{0}\right)\right) \leq \mu \operatorname{meas}\left(Q_{R}^{\lambda, \theta}\right), \quad \mu<N_{4}^{-2},
$$

for some $k$, then

$$
\sup _{Q_{R}^{1, \frac{\theta}{2}}\left(x^{0} ; t^{0}\right)}(u-k) \leq N_{4} \sqrt{\mu} \sup _{Q_{R}^{\lambda, \theta}\left(x^{0} ; t^{0}\right)}(u-k)
$$

(here $\mathrm{N}_{4}$ is the constant from Lemma 3.1).

2. If a nonnegative Lipschitz supersolution of the equation $\mathcal{M} V=0$ in $Q_{R}^{\lambda, \theta}\left(x^{0} ; t^{0}\right)$ satisfies

$$
\operatorname{meas}\left(\{V<k\} \cap Q_{R}^{\lambda, \theta}\left(x^{0} ; t^{0}\right)\right) \leq \mu \operatorname{meas}\left(Q_{R}^{\lambda, \theta}\right), \quad \mu \leq \mu_{1} \equiv\left(2 N_{4}\right)^{-2},
$$

for some $k>0$, then

$$
V \geq \frac{k}{2} \quad \text { in } \quad Q_{R}^{1, \frac{\theta}{2}}\left(x^{0} ; t^{0}\right)
$$

If, moreover, $V$ satisfies

$$
V\left(\cdot ; t^{0}-\theta R^{2}\right) \geq k \quad \text { in } \quad B_{\lambda R}\left(x^{0}\right),
$$

then estimate (44) holds true in $Q_{R}^{1, \theta}\left(x^{0} ; t^{0}\right)$.

Proof. 1. We apply Lemma 3.1 to $u-k$.

2. We apply Lemma 3.1 and Remark 6 to $u=k-V$.

Lemma 3.2. Suppose $\mathcal{M}$ satisfies the assumptions of Lemma 3.1 in $Q_{R}\left(x^{0} ; t^{0}\right)$. For any $\left.\left.\delta_{0} \in\right] 0,1\right]$ there exists $\left.\theta_{0} \in\right] 0,1[$ such that if a nonnegative Lipschitz supersolution of the equation $\mathcal{M} V=0$ in $Q_{R}^{1, \theta_{0}}\left(x^{0} ; t^{0}\right)$ satisfies

$$
\operatorname{meas}\left(\left\{V\left(\cdot ; t^{0}-\theta_{0} R^{2}\right) \geq k\right\} \cap B_{R}\left(x^{0}\right)\right) \geq \delta_{0} \operatorname{meas}\left(B_{R}\right)
$$

for some $k>0$, then

$$
\operatorname{meas}\left(\left\{V(\cdot ; \bar{t}) \geq \frac{\delta_{0}}{3} k\right\} \cap B_{R}\left(x^{0}\right)\right) \geq \frac{\delta_{0}}{3} \operatorname{meas}\left(B_{R}\right)
$$

for any $\bar{t} \in\left[t^{0}-\theta_{0} R^{2}, t^{0}\right]$. Moreover, $\theta_{0}$ is completely determined by $\delta_{0}, n, \nu, q, \ell$, and the quantity $\widehat{\mathcal{N}}$. 
Proof. Without loss of generality, we assume that $\left(x^{0} ; t^{0}\right)=(0 ; 0)$. For a nonnegative test function $\eta$ we have

$$
\int_{Q_{R}^{\lambda, \theta_{0}}}\left(\partial_{t} V \eta+a_{i j} D_{j} V D_{i} \eta+b_{i} D_{i} V \eta\right) d x d t \geq 0
$$

We take $\eta=\chi_{\{t<\bar{t}\}} \cdot(V-k)_{-} \zeta^{2}(x)$, where $\zeta$ is a smooth cut-off function in $B_{R}, \bar{t} \in$ ] $\left.-\theta_{0} R^{2}, 0\right]$. Using (11), (2), and the Young inequality, we obtain

$$
\begin{aligned}
\int_{B_{R}} & \left.(V-k)_{-}^{2} \zeta^{2}\right|^{t=\bar{t}} d x+\nu \int_{Q_{R}^{1, \theta_{0}}} \chi_{\{t<\bar{t}\}}\left|D(V-k)_{-}\right|^{2} \zeta^{2} d x d t \\
\leq & \left.\int_{B_{R}}(V-k)_{-}^{2} \zeta^{2}\right|^{t=-\theta_{0} R^{2}} d x \\
& +\int_{Q_{R}^{1, \theta_{0}}} \chi_{\{t<\bar{t}\}} x\left(C_{13}(n, \nu)(V-k)_{-}^{2}|D \zeta|^{2} d x d t+2 b_{i}(V-k)_{-}^{2} \zeta D_{i} \zeta\right) d x d t
\end{aligned}
$$

Now we choose $\zeta$ such that $\zeta \equiv 1$ in $B_{(1-\sigma) R}$ and $|D \zeta| \leq \frac{2}{\sigma R}$, where $\sigma<1$ is a parameter to be chosen later. Observing that $(V-k)_{-}^{2} \leq k^{2}$, we estimate the right-hand side of (46) by

$$
k^{2}\left[\left(1-\delta_{0}\right) \operatorname{meas}\left(B_{R}\right)+C_{13} \theta_{0} R^{2} \cdot \frac{4 \operatorname{meas}\left(B_{R}\right)}{(\sigma R)^{2}}+\frac{2}{\sigma R}\|\mathbf{b}\|_{q, \ell, Q_{R}}\|\mathbf{1}\|_{q^{\prime}, \ell^{\prime}, Q_{R}^{1, \theta_{0}}}\right] .
$$

On the other hand,

$$
\begin{aligned}
\left.\int_{B_{R}}(V-k)_{-}^{2} \zeta^{2}\right|^{t=\bar{t}} d x & \geq\left.\int_{\left\{V<\frac{\delta_{0}}{3} k\right\} \cap B_{(1-\sigma) R}}(V-k)_{-}^{2}\right|^{t=\bar{t}} d x \\
& \geq\left(1-\frac{\delta_{0}}{3}\right)^{2} k^{2} \operatorname{meas}\left(\left\{V(\cdot ; \bar{t})<\frac{\delta_{0}}{3} k\right\} \cap B_{(1-\sigma) R}\right) .
\end{aligned}
$$

Thus,

$$
\operatorname{meas}\left(\left\{V(\cdot ; \bar{t})<\frac{\delta_{0}}{3} k\right\} \cap B_{(1-\sigma) R}\right) \leq \frac{\operatorname{meas}\left(B_{R}\right)}{\left(1-\frac{\delta_{0}}{3}\right)^{2}} \cdot\left[\left(1-\delta_{0}\right)+\frac{4 C_{13} \theta_{0}}{\sigma^{2}}+\frac{C(n) \theta_{0}^{\frac{2}{\ell^{\prime}}} \widehat{\mathcal{N}}}{\sigma}\right],
$$

whence

$\operatorname{meas}\left(\left\{V(\cdot ; \bar{t})<\frac{\delta_{0}}{3} k\right\} \cap B_{R}\right) \leq \frac{\operatorname{meas}\left(B_{R}\right)}{\left(1-\frac{\delta_{0}}{3}\right)^{2}} \cdot\left[\left(1-\delta_{0}\right)+C(n) \sigma+\frac{4 C_{13} \theta_{0}}{\sigma^{2}}+\frac{C(n) \theta_{0}^{\frac{2}{\ell^{\prime}}} \widehat{\mathcal{N}}}{\sigma}\right]$.

Since $1-\delta_{0} \leq\left(1-\frac{\delta_{0}}{3}\right)^{3}-\frac{8}{27} \delta_{0}^{2}$, we can choose $\sigma$ and then $\theta_{0}$ so small that the right-hand side is not greater that $\left(1-\frac{\delta_{0}}{3}\right)$ meas $\left(B_{R}\right)$, and the lemma follows.

Lemma 3.3. Suppose $\mathcal{M}$ satisfies the assumptions of Lemma 3.1 in $Q_{R}^{\lambda, \theta}\left(x^{0} ; t^{0}\right)$ with $\lambda>1$. Also, suppose that a nonnegative Lipschitz supersolution of the equation $\mathcal{M} V=0$ in $Q_{R}^{\lambda, \theta}\left(x^{0} ; t^{0}\right)$ is such that

$$
\operatorname{meas}\left(\left\{V(\cdot ; t) \geq k_{0}\right\} \cap B_{R}\left(x^{0}\right)\right) \geq \delta_{1} \operatorname{meas}\left(B_{R}\right) \quad \text { for any } t \in\left[t^{0}-\theta R^{2}, t^{0}\right],
$$

for some $k_{0}>0$ and $\delta_{1}>0$. Then for any $\left.\mu \in\right] 0,1[$ there exists $s>1$ such that

$$
\operatorname{meas}\left(\left\{V<2^{-s} k_{0}\right\} \cap Q_{R}^{1, \theta}\left(x^{0} ; t^{0}\right)\right) \leq \mu \operatorname{meas}\left(Q_{R}^{1, \theta}\right) .
$$

Moreover, s is completely determined by $n, \nu, \lambda, \theta, \mu, \delta_{1}, q, \ell$, and the quantity $\widehat{\mathcal{N}}$. 
Proof. Without loss of generality, we assume $\left(x^{0} ; t^{0}\right)=(0 ; 0)$. For $m \in \mathbb{Z}_{+}$, we put $k_{m}=2^{-m} k_{0}$,

$$
\mathcal{E}_{m}(t)=\left\{x \in B_{R}: k_{m+1} \leq V(x, t)<k_{m}\right\}, \quad \mathcal{E}_{m}=\left\{(x ; t): t \in\left[-\theta R^{2}, 0\right], x \in \mathcal{E}_{m}(t)\right\} .
$$

In (45) we take $\eta=\left(V-k_{m}\right)-\zeta^{2}(x)$, where $\zeta$ is a smooth cut-off function vanishing on a neighborhood of $\partial B_{\lambda R}$ and satisfying $\zeta \equiv 1$ in $B_{R},|D \zeta| \leq \frac{2}{(\lambda-1) R}$. As in the proof of Lemma 3.2, we obtain

$$
\int_{\left\{V<k_{m}\right\}}|D V|^{2} \zeta^{2} d x d t=\int_{Q_{R}^{\lambda, \theta}}\left|D\left(V-k_{m}\right)_{-}\right|^{2} \zeta^{2} d x d t \leq C_{14}(n, \nu, \lambda, \theta, \ell, \widehat{\mathcal{N}}) k_{m}^{2} R^{n}
$$

Next, De Giorgi's inequality (see, e.g., [LSU, Chapter II, (5.6)]) and the assumption (47) imply that

$$
\left(k_{m}-k_{m+1}\right) \cdot \operatorname{meas}\left(\left\{V(\cdot ; t)<k_{m+1}\right\} \cap B_{R}\right) \leq \frac{C(n) R}{\delta_{1}} \int_{\mathcal{E}_{m}(t)}|D V(\cdot ; t)| d x, t \in\left[-\theta R^{2}, 0\right] .
$$

We integrate this relation with respect to $t$ and then square both parts, arriving at

$$
k_{m+1}^{2} \operatorname{meas}^{2}\left(\left\{V<k_{m+1}\right\} \cap Q_{R}^{1, \theta}\right) \leq \frac{C(n) R^{2}}{\delta_{1}^{2}} \int_{\mathcal{E}_{m}}|D V|^{2} d x d t \cdot \operatorname{meas}\left(\mathcal{E}_{m}\right) .
$$

Together with (48), this gives

$$
\operatorname{meas}^{2}\left(\left\{V<k_{m+1}\right\} \cap Q_{R}^{1, \theta}\right) \leq C(n) C_{14} \delta_{1}^{-2} R^{n+2} \cdot \operatorname{meas}\left(\mathcal{E}_{m}\right) .
$$

Therefore,

$$
\begin{aligned}
s \cdot \operatorname{meas}^{2}\left(\left\{V<k_{s}\right\} \cap\right. & \left.Q_{R}^{1, \theta}\right) \leq \sum_{m=0}^{s-1} \operatorname{meas}^{2}\left(\left\{V<k_{m+1}\right\} \cap Q_{R}^{1, \theta}\right) \\
& \leq C_{15} \delta_{1}^{-2} \cdot \operatorname{meas}\left(Q_{R}^{1, \theta}\right) \cdot \sum_{m=0}^{s-1} \operatorname{meas}\left(\mathcal{E}_{m}\right) \leq C_{15} \delta_{1}^{-2} \cdot \operatorname{meas}^{2}\left(Q_{R}^{1, \theta}\right)
\end{aligned}
$$

(here $C_{15}$ depends on the same quantities as $C_{14}$ ), and the claim follows.

Corollary 3.2. Suppose $\mathcal{M}$ satisfies the assumptions of Lemma 3.1 in $Q_{R}^{2,1}$.

1. If a nonnegative Lipschitz supersolution of the equation $\mathcal{M} V=0$ in $Q_{R}^{2,1}$ satisfies

$$
\operatorname{meas}\left(\{V(\cdot ; \bar{t}) \geq k\} \cap B_{R}\right) \geq \delta \operatorname{meas}\left(B_{R}\right)
$$

for some $\left.\left.\bar{t} \in\left[-R^{2},-\Theta R^{2}\right], k>0, \delta, \Theta \in\right] 0,1\right]$, then

$$
V \geq \beta_{1} k \quad \text { in } \quad Q_{R}^{1, \frac{\theta_{1}}{2}}\left(0 ; \bar{t}+\theta_{1} R^{2}\right) .
$$

Here $\theta_{1}=\min \left\{\Theta, \theta_{0}\right\}$, where $\theta_{0}=\theta_{0}(\delta, n, \nu, q, \ell, \widehat{\mathcal{N}})$ is the constant from Lemma 3.2 , while $\beta_{1}$ depends on the same quantities as $\theta_{0}$.

2. If relation (49) holds true with $\delta=1$, i.e.,

$$
V(\cdot ; \bar{t}) \geq k \quad \text { in } \quad B_{R},
$$

then for any $\sigma \in] 0,1[$ we have

$$
V \geq \beta_{1} k \quad \text { in } \quad Q_{R}^{\sigma, \theta_{1}}\left(0 ; \bar{t}+\theta_{1} R^{2}\right) .
$$

In this case, $\beta_{1}$ depends also on $\sigma$. 
Proof. First, we use Lemma 3.2 with $t^{0}=\bar{t}+\theta_{1} R^{2}$. Then, in case 1, we apply Lemma 3.3 with $R \mapsto \frac{3}{2} R, \lambda=\frac{4}{3}, \theta=\frac{4}{9} \theta_{1}, \delta_{1}=\frac{2^{n} \delta}{3^{n+1}}, k_{0}=\frac{\delta}{3} k$, and $\mu=\mu_{1}$, where $\mu_{1}=$ $\mu_{1}\left(n, \nu, \frac{3}{2}, \theta_{1}, q, \ell, \widehat{\mathcal{N}}\right)$ is the constant from part 2 of Corollary 3.1. Finally, Corollary 3.1 with $\lambda=\frac{3}{2}$ and $\theta=\theta_{1}$ gives (50) with $\beta_{1}=\frac{\delta}{3 \cdot 2^{s+1}}$, where $s=s\left(n, \nu, \frac{3}{2}, \theta_{1}, \mu_{1}, \delta_{1}, q, \ell, \widehat{\mathcal{N}}\right)$ is the constant from Lemma 3.3.

In case 2, we apply Lemma 3.3 with $\lambda=2, \theta=\theta_{1}, \delta_{1}=\frac{\delta}{3}, k_{0}=\frac{\delta}{3} k$, and $\mu=$ $\mu_{1}\left(n, \nu, \sigma^{-1}, \sigma^{-2} \theta_{1}, q, \ell, \widehat{\mathcal{N}}\right)$. Now, the last claim of Corollary 3.1 with $R \mapsto \sigma R, \lambda=\sigma^{-1}$ and $\theta=\sigma^{-2} \theta_{1}$ gives (52) with $\beta_{1}=\frac{\delta}{3 \cdot 2^{s+1}}$, where

$$
s=s\left(n, \nu, \sigma^{-1}, \sigma^{-2} \theta_{1}, \mu_{1}, \delta_{1}, q, \ell, \widehat{\mathcal{N}}\right) .
$$

Lemma 3.4. Suppose $\mathcal{M}$ satisfies the assumptions of Lemma 3.1 in $Q_{R}^{2,1}$. Also, suppose that a nonnegative Lipschitz supersolution of the equation $\mathcal{M} V=0$ in $Q_{R}^{2,1}$ satisfies (51) for some $k>0$ and $\left.\left.\bar{t} \in\left[-R^{2},-\Theta R^{2}\right], \Theta \in\right] 0,1\right]$. Then

$$
V \geq \beta_{2} k \quad \text { in } \quad \widehat{Q}=B_{\frac{R}{2}} \times[\bar{t}, 0] .
$$

Moreover, $\beta_{2}$ is completely determined by $\Theta, n, \nu, q, \ell$, and the quantity $\widehat{\mathcal{N}}$.

Proof. Setting $M=\operatorname{entier}\left(\frac{|\bar{t}|}{\theta_{1} R^{2}}\right)+1$ and $\widehat{\theta}_{1}=\frac{|\bar{t}|}{M R^{2}}$, we consider the cylinders

$$
Q^{(m)}=Q_{R}^{1-\frac{m}{2 M}, \widehat{\theta}_{1}}\left(0 ; \bar{t}+m \widehat{\theta}_{1} R^{2}\right), \quad m=1, \ldots, M .
$$

By (52), we obtain consecutively

$$
V \geq \widehat{\beta}_{1} \cdot \inf _{Q^{(m)}} V \quad \text { in } \quad Q^{(m+1)}
$$

where $\widehat{\beta}_{1}=\widehat{\beta}_{1}(n, \Theta, \nu, q, \ell, \widehat{\mathcal{N}})>0$.

Since $\widehat{Q} \subset \bigcup_{m} Q^{(m)}$, this ensures (53) with $\beta_{2}=\widehat{\beta}_{1}^{M}$.

Corollary 3.3. Suppose $\mathcal{M}$ satisfies the assumptions of Lemma 3.1 in $Q_{R}^{2,1}$. If a nonnegative Lipschitz supersolution of the equation $\mathcal{M V}=0$ in $Q_{R}^{2,1}$ satisfies (49) for some $k>0, \delta \in] 0,1]$, and $\bar{t} \in\left[-R^{2},-\frac{3}{4} R^{2}\right]$, then

$$
V \geq \beta_{3} k \quad \text { in } \quad Q_{\frac{R}{2}}
$$

where $\beta_{3}$ is completely determined by $\delta, n, \nu, q, \ell$, and the quantity $\widehat{\mathcal{N}}$.

Proof. It suffices to apply part 1 of Corollary 3.2 and then Lemma 3.4.

Corollary 3.4. Suppose $\mathcal{M}$ satisfies the assumptions of Lemma 3.1 in $Q_{R}^{2,1}$. If a nonnegative Lipschitz supersolution of the equation $\mathcal{M V}=0$ in $Q_{R}^{2,1}$ satisfies

$$
\operatorname{meas}\left(\{V>k\} \cap Q_{R}\right) \geq \widehat{\delta} \operatorname{meas}\left(Q_{R}\right)
$$

for some $k>0, \widehat{\delta} \in] 0,1]$, then

$$
V \geq \beta_{4} k \quad \text { in } \quad Q_{R}^{\frac{1}{2}, \frac{\widehat{\delta}}{4}},
$$

where $\beta_{4}$ is completely determined by $\widehat{\delta}, n, \nu, q, \ell$, and the quantity $\widehat{\mathcal{N}}$.

Proof. Obviously, inequality (55) implies

$$
\operatorname{meas}\left(\{V>k\} \cap Q_{R}^{1,1-\frac{\widehat{\delta}}{2}}\left(0 ;-\frac{\widehat{\delta}}{2} R^{2}\right)\right) \geq \frac{\widehat{\delta}}{2} \operatorname{meas}\left(Q_{R}\right) .
$$

Therefore, there exists $\bar{t} \in\left[-R^{2},-\frac{\widehat{\delta}}{2} R^{2}\right]$ such that (49) is true with $\delta=\frac{\widehat{\delta}}{2}$. By part 1 of Corollary 3.2, $V\left(\cdot ; \bar{t}+\frac{\theta_{1}}{2} R^{2}\right) \geq \beta_{1} k$ in $B_{R}$. Finally, we observe that $\bar{t}+\frac{\theta_{1}}{2} R^{2} \leq-\frac{\widehat{\delta}}{4} R^{2}$, and Lemma 3.4 provides (56). 
Corollary 3.5 (strong maximum principle). Suppose $\mathcal{M}$ satisfies the assumption of Lemma 3.1 in $Q$. Then any nonconstant Lipschitz supersolution of the equation $\mathcal{M V}=0$ in $Q$ cannot attain its minimum at a point of $\partial Q \backslash \partial^{\prime} Q$.

Proof. There is no loss of generality in assuming that $\inf _{Q} V=0$.

Suppose the claim is not true. Then there exists $\left(x^{0} ; t^{0}\right) \in \bar{Q} \backslash \partial^{\prime} Q$ such that $V\left(x^{0} ; t^{0}\right)=$ 0 , but $V \not \equiv 0$ in $Q_{R}\left(x^{0} ; t^{0}\right) \subset Q_{R}^{2,1}\left(x^{0} ; t^{0}\right) \subset Q$ with some $R$. Then relation (55) is valid for some $k>0$ and $\delta>0$, and we obtain (56), a contradiction.

Lemma 3.5. Suppose $\mathcal{M}$ satisfies the assumptions of Lemma 3.1 in $Q_{2 R}$. Then any Lipschitz solution of the equation $\mathcal{M} u=0$ in $Q_{2 R}$ satisfies the estimate

$$
\underset{Q_{\frac{R}{2}}}{\operatorname{OSc}} u \leq \varkappa_{1} \underset{Q_{2 R}}{\operatorname{OSc}} u
$$

where $\varkappa_{1}<1$ depends on $n, \nu, q, \ell$, and the quantity $\widehat{\mathcal{N}}$.

Proof. We set $k=\frac{1}{2} \operatorname{osc}_{Q_{2 R}} u$ and consider two functions $V_{1}=u-\inf _{Q_{2 R}} u$ and $V_{2}=$ $\sup _{Q_{2 R}} u-u$. At least one of them satisfies (49) with $\delta=\frac{1}{2}$ and $\bar{t}=-R^{2}$. Therefore, for this function Corollary 3.3 gives estimate (54), which implies (57) with $\varkappa_{1}=1-$ $\frac{1}{2} \beta_{3}\left(\frac{1}{2}, n, \nu, q, \ell, \widehat{\mathcal{N}}\right)$.

Corollary 3.6 (Hölder estimate). Suppose $\mathcal{M}$ satisfies the assumptions of Lemma 3.1 in $Q_{R_{0}}$. Also, let $\sup _{R<R_{0}} \widehat{\mathcal{N}}(R, 1,1)<\infty$. Then any Lipschitz solution of the equation $\mathcal{M} u=0$ in $Q_{R_{0}}$ satisfies the estimate

$$
\underset{Q_{\rho}}{\operatorname{osc}} u \leq N_{5}\left(\frac{\rho}{r}\right)^{\gamma_{1}} \cdot \underset{Q_{r}}{\operatorname{osc}} u, \quad 0<\rho<r \leq R_{0}
$$

where $N_{5}$ and $\gamma_{1}$ depend on $n, \nu, q$, and $\sup _{R<R_{0}} \widehat{\mathcal{N}}(R, 1,1)$.

Proof. Iteration of (57) gives (58) with $\gamma_{1}=-\log _{4}\left(\varkappa_{1}\right)$.

Corollary 3.7 (the Liouville theorem). Let $\mathcal{M}$ be an operator of the form (DP) in $\mathbb{R}^{n} \times \mathbb{R}_{-}$, and let conditions (11) and (2) be satisfied. Also, let $\mathbf{b} \in L_{q, \ell, \text { loc }}\left(\mathbb{R}^{n} \times \mathbb{R}_{-}\right)$with some $q$ and $\ell$ satisfying (31) ( $q$ and/or $\ell$ may be infinite). Finally, assume that

$$
\liminf _{R \rightarrow \infty} \widehat{\mathcal{N}}(R, 1,1)<\infty .
$$

Then any bounded Lipschitz solution of the equation $\mathcal{M} u=0$ in $\mathbb{R}^{n} \times \mathbb{R}_{-}$is constant.

Remark 7. If $\mathbf{b} \in L_{q, \ell}\left(\mathbb{R}^{n} \times \mathbb{R}_{-}\right)$, then, obviously, (59) is fulfilled.

Proof. Iteration of (57) with respect to a suitable sequence $R_{m} \rightarrow \infty$ gives the claim.

To prove the Harnack inequality, we need the following modification of Lemma 3.4.

Lemma 3.4' (slant cylinder). Suppose $\mathcal{M}$ satisfies the assumptions of Lemma 3.1 in $Q_{R}^{\lambda, 1}, \lambda>2$. Also, suppose that a nonnegative Lipschitz supersolution of the equation $\mathcal{M} V=0$ in $Q_{R}^{\lambda, 1}$ satisfies

$$
V\left(\cdot ;-R^{2}\right) \geq k \quad \text { in } \quad B_{R}\left(x^{0}\right)
$$

for some $k>0$ and $x^{0} \in B_{(\lambda-2) R}$. Then for any $x^{1} \in B_{(\lambda-2) R}$ we have

$$
V \geq \widehat{\beta}_{2} k \quad \text { in } \quad \widetilde{Q}=\left\{(x ; t): t \in\left[-R^{2}, 0\right], x \in B_{\frac{R}{2}}\left(x^{1}+\left(x^{1}-x^{0}\right) \frac{t}{R^{2}}\right)\right\} .
$$

Moreover, $\widehat{\beta}_{2}$ is completely determined by $\lambda, n, \nu, q, \ell$, and the quantity $\widehat{\mathcal{N}}$. 
Proof. We put $\widehat{x}(t)=x^{1}+\left(x^{1}-x^{0}\right) \frac{t}{R^{2}}$. Then it is easily seen that the function $\tilde{V}(x ; t)=$ $V(x-\widehat{x}(t) ; t)$ is a nonnegative Lipschitz supersolution of the equation

$$
\widetilde{\mathcal{M}} V \equiv \partial_{t} V-D_{i}\left(\widetilde{a}_{i j}(x ; t) D_{j} V\right)+\widetilde{b}_{i}(x ; t) D_{i} V=0
$$

in $Q_{R}^{2,1}$, where

$$
\tilde{a}_{i j}(x ; t)=a_{i j}(x-\widehat{x}(t) ; t) ; \quad \widetilde{b}_{i}(x ; t)=b_{i}(x-\widehat{x}(t) ; t)+\frac{x_{i}^{1}-x_{i}^{0}}{R^{2}} .
$$

Note that $\widetilde{\mathcal{M}}$ satisfies the assumptions of Lemma 3.1 in $Q_{R}^{2,1}$, and the quantity $\widetilde{\widehat{\mathcal{N}}}$ is bounded by $\widehat{\mathcal{N}}+C_{16}(\lambda, n)$. Using Lemma 3.4 (with $\Theta=1$ ), we obtain (60).

The next statement is a parabolic analog of Lemma 2.4. For $(x ; t) \in Q$, we introduce the notation $d_{\text {par }}\left((x ; t), \partial^{\prime} Q\right)=\inf \left\{\rho>0: Q_{\rho}(x ; t) \subset Q\right\}$.

Lemma 3.6. Suppose $\mathcal{M}$ satisfies the assumptions of Lemma 3.1 in $Q_{2 R}$, and let $\mathbf{b} \in$ $\mathbb{M}_{q, \ell}^{\alpha}\left(Q_{2 R}\right)$. Also, suppose that, for a nonnegative Lipschitz supersolution of the equation $\mathcal{M} V=0$ in $Q_{2 R}$ and some $(y ; s) \in Q_{R}^{2,2}\left(0 ;-2 R^{2}\right)$, the inequality $\inf _{B_{\rho}(y)} V(\cdot ; s)=k>0$ is fulfilled with $\rho=\frac{1}{4} d_{\operatorname{par}}\left((y ; s), \partial^{\prime} Q_{2 R}\right)$. Then

$$
\inf _{Q_{R}} V \geq N_{6}\left(\frac{\rho}{R}\right)^{\widehat{\gamma}_{1}} k
$$

where $N_{6}$ and $\widehat{\gamma}_{1}$ depend on $n, \nu, q, \ell$, and $\|\mathbf{b}\|_{\mathbb{M}_{q, \ell}^{\alpha}\left(Q_{2 R}\right)}$.

Proof. We denote by $\mathfrak{N}$ the integer such that $2^{-(\mathfrak{N}+1)} R \leq \rho<2^{-\mathfrak{N}} R$ and introduce a sequence of cylinders $Q_{\mathfrak{r}_{m}}^{4,1}\left(y^{m} ; t^{m}\right), m=0, \ldots, \mathfrak{N}$, as follows:

$$
\begin{array}{lll}
\mathfrak{r}_{0}=2^{-(\mathfrak{N}+1)} R, & y^{0}=y, & t^{0}=s+\mathfrak{r}_{0}^{2} ; \\
\mathfrak{r}_{m}=2 \mathfrak{r}_{m-1}, & y^{m}=y^{m-1}-\min \left\{2 \mathfrak{r}_{m},\left|y^{m-1}\right|\right\} \mathbf{e}, & t^{m}=t^{m-1}+\mathfrak{r}_{m}^{2}
\end{array}
$$

(here $\mathbf{e}=\frac{y}{|y|}$ ). Also we denote $y^{-1}=y, t^{-1}=s$.

Direct computations show that $Q_{\mathbf{r}_{m}}^{4,1}\left(y^{m} ; t^{m}\right) \subset Q_{2 R}$ for all $m=0, \ldots, \mathfrak{N}$. Therefore, the assumptions of Lemma $3.4^{\prime}$ (with $\lambda=4, x^{0}=y^{m-1}$ ) are fulfilled in $Q_{\mathfrak{r}_{m}}^{4,1}\left(y^{m} ; t^{m}\right)$. Using Lemma $3.4^{\prime}$ with $x^{1} \in B_{2 \mathfrak{r}_{m}}\left(y^{m}\right)$, we obtain the inequality

$$
V\left(\cdot ; t^{m}\right) \geq \widehat{\beta}_{2}\left(4, n, \nu, q, \ell,\|\mathbf{b}\|_{\mathbb{M}_{q, \ell}^{\alpha}\left(Q_{2 R}\right)}\right) \cdot \inf _{B_{\mathbf{r}_{m}}\left(y^{m-1}\right)} V\left(\cdot ; t^{m-1}\right) \quad \text { in } \quad B_{\frac{5}{2}} \mathfrak{r}_{m}\left(y^{m}\right),
$$

and, in particular,

$$
\inf _{B_{\mathbf{r}_{m+1}}\left(y^{m}\right)} V\left(\cdot ; t^{m}\right) \geq \widehat{\beta}_{2} \cdot \inf _{B_{\mathfrak{r}_{m}}\left(y^{m-1}\right)} V\left(\cdot ; t^{m-1}\right) .
$$

Since $\mathfrak{r}_{\mathfrak{N}+1}=R$ and $y^{\mathfrak{N}}=0$, we obtain

$$
\inf _{B_{R}} V\left(\cdot ; t^{\mathfrak{N}}\right) \geq \widehat{\beta}_{2}^{\mathfrak{N}+1} \cdot \inf _{B_{\mathfrak{r}_{0}}(y)} V(\cdot ; s) \geq\left(\frac{\rho}{2 R}\right)^{\widehat{\gamma}_{1}} \cdot k
$$

where $\widehat{\gamma}_{1}=-\log _{2}\left(\widehat{\beta}_{2}\right)$.

It is easy to estimate $t^{\mathfrak{N}}$ :

$$
t^{\mathfrak{N}}=s+\sum_{m=0}^{\mathfrak{N}} \mathfrak{r}_{m}^{2}=s+\mathfrak{r}_{0}^{2} \cdot \frac{2^{2 \mathfrak{N}+2}-1}{3}<s+\frac{R^{2}}{3} \leq-\frac{5}{3} R^{2} .
$$


Now we use Lemma $3.4^{\prime}$ (with $\lambda=4, x^{0} \in B_{\frac{R}{2}}, x^{1} \in B_{R}$ ) in $Q_{\frac{R}{2}}^{4,1}\left(0 ; t^{\mathfrak{N}}+\frac{R^{2}}{4}\right)$. Since the slant cylinders for $x^{0} \in B_{\frac{R}{2}}, x^{1} \in B_{R} \operatorname{cover} Q_{R}^{1, \frac{1}{8}}\left(0 ; t^{\mathfrak{N}}+\frac{R^{2}}{4}\right)$, we obtain

$$
\inf _{Q_{R}^{1, \frac{1}{8}}}\left(0 ; t^{\mathfrak{N}}+\frac{R^{2}}{4}\right) V \geq \widehat{\beta}_{2}\left(\frac{\rho}{2 R}\right)^{\widehat{\gamma}_{1}} \cdot k .
$$

Repeating this process, we cover $Q_{R}$. This gives (61) with $N_{6}=2^{-\widehat{\gamma}_{1}} \widehat{\beta}_{2}^{31}$.

Theorem 3.7 (the Harnack inequality). Let $\mathcal{M}$ be an operator of the form (DP) in $Q_{2 R}$, and let conditions (11) and (2) be satisfied. Also, let $\mathbf{b} \in \mathbb{M}_{q, \ell}^{\alpha}\left(Q_{2 R}\right)$ with some $q$ and $\ell$ satisfying (31) ( $q$ and/or $\ell$ may be infinite).

Then there exists a positive constant $N_{7}$ depending on $n, \nu, q$, $\ell$, and $\|\mathbf{b}\|_{\mathbb{M}_{q, \ell}^{\alpha}\left(Q_{2 R}\right)}$, such that any nonnegative Lipschitz solution of the equation $\mathcal{M} u=0$ in $Q_{2 R}$ satisfies

$$
\sup _{Q_{R}\left(0 ;-2 R^{2}\right)} u \leq N_{7} \cdot \inf _{Q_{R}} u \text {. }
$$

Proof. As in Theorem 2.5, we denote by $(y ; s)$ a maximum point of the function

$$
v(x ; t)=\left(d_{\operatorname{par}}\left((x ; t), \partial^{\prime} Q_{2 R}\right)\right)^{\widehat{\gamma}_{1}} \cdot u(x ; t) ; \quad(x ; t) \in Q_{R}^{2,2}\left(0 ;-2 R^{2}\right)
$$

(here $\widehat{\gamma}_{1}$ is the constant from Lemma 3.6) and set

$$
\rho=\frac{1}{4} d_{\operatorname{par}}\left((y ; s), \partial^{\prime} Q_{2 R}\right) ; \quad \mathfrak{M}=v(y ; s)=(4 \rho)^{\widehat{\gamma}_{1}} \cdot u(y ; s) .
$$

Obviously,

$$
\begin{aligned}
\sup _{Q_{R}\left(0 ;-2 R^{2}\right)} u & \leq \frac{\mathfrak{M}}{R \widehat{\gamma}_{1}}=\left(\frac{4 \rho}{R}\right)^{\widehat{\gamma}_{1}} \cdot u(y ; s) ; \\
\sup _{Q_{2 \rho}(y ; s)} u & \leq \frac{\mathfrak{M}}{(2 \rho) \widehat{\gamma}_{1}}=2^{\widehat{\gamma}_{1}} \cdot u(y ; s) .
\end{aligned}
$$

Denote $k=\frac{1}{2} u(y ; s)$. If meas $\left(\{u>k\} \cap Q_{2 \rho}(y ; s)\right) \leq \mu$ meas $\left(Q_{2 \rho}\right)$, then part 1 of Corollary 3.1 (with $\lambda=2$ and $\theta=4$ ) and (64) imply the relation

$$
k=u(y ; s)-k \leq \sup _{Q_{\rho}(y ; s)}(u-k) \leq N_{4} \sqrt{\mu} \sup _{Q_{2 \rho}(y ; s)}(u-k) \leq N_{4} \sqrt{\mu}\left(2^{\gamma_{1}+1}-1\right) k,
$$

which is impossible for $\mu \leq \mu_{2} \equiv \frac{1}{2^{2 \gamma_{1}+2}} N_{4}^{-2}$. Thus, meas $\left(\{u>k\} \cap Q_{2 \rho}(y ; s)\right) \geq$ $\mu_{2}$ meas $\left(Q_{2 \rho}\right)$, and Corollary 3.4 (with $\widehat{\delta}=\mu_{2}$ ) gives

$$
\inf _{B_{\rho}(y)} u(\cdot ; s) \geq \beta_{4} k=\frac{\beta_{4}}{2} \cdot u(y ; s) .
$$

Finally, Lemma 3.6 shows that

$$
\inf _{Q_{R}} u \geq N_{6}\left(\frac{\rho}{R}\right)^{\widehat{\gamma}_{1}} \inf _{B_{\rho}(y)} u(\cdot ; s) .
$$

Combining (63), (65), and (66), we arrive at (62) with $N_{7}=\frac{2^{2 \widehat{\gamma}_{1}+1}}{\beta_{4} N_{6}}$.

As in $\S 2$, the proofs of Lemmas 3.1-3.3 run without changes also for the weak sub/supersolutions of the equation $\mathcal{M} u=0$ if the bilinear form

$$
\widehat{\mathcal{B}}\langle u, \eta\rangle \equiv \int_{Q_{R}^{\lambda, \theta}} b_{i} D_{i} u \eta d x d t
$$


extends by continuity to the pairs $\left(v, v \zeta^{2}\right)$ with $v \in \mathcal{V}\left(Q_{R}^{\lambda, \theta}\right)$. Unfortunately, we have no parabolic analog of the sharp results by Maz'ya-Verbitskir; we can give only rather rough sufficient conditions. The simplest one is

$$
\mathbf{b} \in \mathbb{M}_{q, \infty}^{\frac{n}{q}-1}\left(Q_{R}^{\lambda, \theta}\right), \quad \frac{n}{2}<q \leq n, \quad \operatorname{div}(\mathbf{b})=0 .
$$

Once Lemmas 3.1-3.3 are proved, all subsequent statements obviously remain valid.

Now, we consider the case where $\alpha(q, \ell)=0$, i.e., $\frac{n}{q}+\frac{2}{\ell}=1$. As in the elliptic case, the main results of this section hold true for the weak (sub/super)solutions without the assumption (2) 5 . The only exceptional situation is $q=n$, where the assumption (2) seems to be unavoidable without smallness restriction on $\mathbf{b} 6$. We explain briefly the changes needed in the proofs.

Like Lemma 2.1, Lemma 3.1 in this case can be proved under the additional assumption $\|\mathbf{b}\|_{q, \ell, Q_{R}^{\lambda, \theta}} \leq \varepsilon(n, \nu)$.

The proofs of Lemmas 3.2 and 3.3 require no changes. Therefore, all subsequent claims hold true whenever $\mathbf{b}$ is sufficiently small.

In what follows we shall assume that $q>n$. Then, as in the elliptic case, the strong maximum principle is valid without any smallness assumption on $\mathbf{b}$.

When proving the Harnack inequality, one can exclude the smallness assumption on $\mathbf{b}$, as in Theorem $2.5^{\prime}$. The result reads as follows.

Theorem 3.7' (the Harnack inequality). Let $\mathcal{M}$ be an operator of the form (DE) in $Q_{2 R}$, and let condition (1) be satisfied. Also, suppose that

$$
\|\mathbf{b}\|_{q, \ell, Q_{2 R}} \leq \mathfrak{B}, \quad \frac{n}{q}+\frac{2}{\ell}=1, \quad q>n .
$$

Then there exists a positive constant $N_{7}^{\prime}$ depending only on $n, \nu, q$, and $\mathfrak{B}$ such that any nonnegative weak solution of the equation $\mathcal{M} u=0$ in $Q_{2 R}$ satisfies

$$
\sup _{Q_{R}\left(0 ;-2 R^{2}\right)} u \leq N_{7}^{\prime} \cdot \inf _{Q_{R}} u
$$

As in the elliptic case, we have two possibilities to prove Hölder estimates for solutions. The first is to take $R$ in Lemma 3.5 so small that the smallness assumptions on $\mathbf{b}$ are satisfied in $Q_{2 R}$. This gives estimate (58) with $\gamma_{1}$ depending only on $n, \nu$, and $q$, while $N_{5}$ depends also on the modulus of continuity of $\mathbf{b}$ in $L_{q, \ell}\left(Q_{R_{0}}\right)$. The second possibility is to use Theorem $3.7^{\prime}$. This gives (58) with $\gamma_{1}$ and $N_{5}$ depending on $n, \nu, q$, and $\|\mathbf{b}\|_{q, \ell, Q_{R_{0}}}$.

Finally, the next statement directly follows from the second version of the Hölder estimate.

Corollary $3.7^{\prime}$ (the Liouville theorem). Let $\mathcal{M}$ be an operator of the form (DP) in $\mathbb{R}^{n} \times \mathbb{R}_{-}$, and let conditions (1) be satisfied. Also, let $\mathbf{b} \in L_{q, \ell}\left(\mathbb{R}^{n} \times \mathbb{R}_{-}\right)$with some $q$ and $\ell$ such that $\frac{n}{q}+\frac{2}{\ell}=1, q>n$. Then any bounded weak solution of the equation $\mathcal{M} u=0$ in $\mathbb{R}^{n} \times \mathbb{R}_{-}$is constant.

\footnotetext{
${ }^{5}$ Also, the assumptions on $\mathbf{b}$ can be relaxed in the scale of Lorentz spaces.

${ }^{6}$ By the Hölder inequality, under the assumption (2), the case where $q=n, \ell=\infty$ is simply included into the case where $q=n, 2<\ell<\infty$.
} 


\section{§4. Applichtion to a problem of hydrodynamics}

In the study of axisymmetric flows of viscous incompressible liquid, the following equation of the (DP) form arises:

$$
\mathcal{M} u \equiv \partial_{t} u-\Delta u+b_{i}\left(x^{\prime}, x_{3} ; t\right) D_{i} u=0 \quad \text { in } \quad \mathbb{R}^{3} \times \mathbb{R}_{-} .
$$

Here we denote $x^{\prime}=\left(x_{1}, x_{2}\right)$, and

$$
\mathbf{b}=\mathbf{v}+\widehat{\mathbf{b}}=\left(v^{1}+\varepsilon \frac{2 x_{1}}{\left|x^{\prime}\right|^{2}}, v^{2}+\varepsilon \frac{2 x_{2}}{\left|x^{\prime}\right|^{2}}, v^{3}\right),
$$

where $\mathbf{v}=\left(v^{1}, v^{2}, v^{3}\right)$ is a solution of the Navier-Stokes system (NSE), and $\varepsilon= \pm 1$.

Namely, see KNSS, the function $u=v_{2} x_{1}-v_{1} x_{2} \equiv\left|x^{\prime}\right| v_{\vartheta}$ satisfies equation (67) with $\varepsilon=+1$ (here $v_{\vartheta}$ is the angular component of the velocity). Next, if $v_{\vartheta}=0$, then the function $u=\left|x^{\prime}\right|^{-2}\left((\operatorname{curl}(\mathbf{v}))_{2} x_{1}-(\operatorname{curl}(\mathbf{v}))_{1} x_{2}\right)$ satisfies equation (67) with $\varepsilon=-1$.

Since $\operatorname{div}(\mathbf{v})=0$ by the NSE, it is easily seen that

$$
\operatorname{div}(\mathbf{b})=4 \pi \varepsilon \delta_{\Gamma}, \quad \Gamma=\left\{\left|x^{\prime}\right|=0\right\} .
$$

Thus, if $\varepsilon=-1$, then the results of $\S 3$ can be applied to (67)- Specifically, we are interested in the Liouville theorem.

Note that $\widehat{\mathbf{b}}$ belongs to $L_{q, \infty, \text { loc }}\left(\mathbb{R}^{3} \times \mathbb{R}_{-}\right)$with any $q<2$, and moreover, satisfies assumption (59) with $q \in] \frac{3}{2}, 2[, \ell=\infty$. Therefore, taking Remark 5 into account, we obtain the following result.

Theorem 4.1. Let $\mathbf{v}$ be an axisymmetric solution of the Navier-Stokes system in $\mathbb{R}^{3} \times$ $\mathbb{R}_{-}$. Also, suppose that $\mathbf{v}$ satisfies (59) with some $q$ and $\ell$ such that $\alpha \equiv \frac{3}{q}+\frac{2}{\ell}-1 \in[0,1[$. Then any bounded Lipschitz solution of (67)-68) with $\varepsilon=-1$ in $\mathbb{R}^{n} \times \mathbb{R}_{-}$is constant.

Remark 8. The assumption (59) is satisfied, for example, if $\mathbf{v}$ obeys the estimate

$$
\left|\mathbf{v}\left(x^{\prime}, x_{3} ; t\right)\right| \leq \frac{C}{\left|x^{\prime}\right|}
$$

(in this case one can take $q \in] \frac{3}{2}, 2[, \ell=\infty$ ), or if

$$
\left|\mathbf{v}\left(x^{\prime}, x_{3} ; t\right)\right| \leq \frac{C}{(-t)^{\frac{1}{2}}}
$$

(in this case it suffices to take $q=\infty, \ell \in] 1,2[$ ).

To deal with the more complicated case of $\varepsilon=+1$, we need the following observation.

Remark 9. The claim of Lemma 3.1 holds true without assumption (2) if $u \leq 0$ on the set $\mathcal{F}=\operatorname{supp}(\operatorname{div}(\mathbf{b}))_{+}$. Similarly, Lemmas 3.2-3.4 and Corollaries 3.1 (part 2), 3.2, 3.4 hold true if $V \geq k$ in $\mathcal{F}$. Lemma 3.3 holds true if $V \geq k_{0}$ in $\mathcal{F}$.

Now we prove the following version of Corollary 3.3.

Lemma 4.2. Let $\mathbf{v}$ be an axisymmetric solution of the Navier-Stokes system in $Q_{R}^{2,1}$, and let $\mathbf{v} \in L_{q, \ell}\left(Q_{R}^{2,1}\right)$ with some $q$ and $\ell$ such that $\alpha \equiv \frac{3}{q}+\frac{2}{\ell}-1 \in[0,1[$. Let $V$ be a nonnegative Lipschitz supersolution of (67)- (68) with $\varepsilon=+1$ in $Q_{R}^{2,1}$. If

$$
\left.V\right|_{\Gamma \cap Q_{R}^{2,1}} \geq k, \quad V \leq \mathfrak{N} k \quad \text { in } Q_{R}^{2,1}
$$

for some $k>0$ and $\mathfrak{N}>1$, then

$$
V \geq \widehat{\beta}_{3} k \quad \text { in } \quad Q_{\frac{R}{2}}
$$

where $\widehat{\beta}_{3}$ is completely determined by $q, \ell, \mathfrak{N}$, and the quantity $\widehat{\mathcal{N}}=R^{-\alpha}\|\mathbf{v}\|_{q, \ell, Q_{R}^{2,1}}$. 
Proof. Putting

$$
\widehat{\mathcal{E}}_{\varkappa}(t)=\left\{x \in B_{R}: V(x, t)>\varkappa k\right\}, \quad \widehat{\mathcal{E}}_{\varkappa}=\left\{(x ; t): t \in\left[-R^{2},-\frac{3}{4} R^{2}\right], x \in \widehat{\mathcal{E}}_{\varkappa}(t)\right\},
$$

we claim that

$$
\operatorname{meas}\left(\widehat{\mathcal{E}}_{\varkappa}\right) \geq \delta \operatorname{meas}\left(Q_{R}^{1, \frac{1}{4}}\right)
$$

for some $\varkappa>0$ and $\delta>0$ depending only on $q, \ell, \mathfrak{N}$, and $\widehat{\mathcal{N}}$.

Indeed, by (69), for any Lipschitz test function $\eta \geq 0$ we obtain

$$
\int_{Q_{R}^{1, \frac{1}{4}}\left(0 ;-\frac{3}{4} R^{2}\right)}\left(\partial_{t} V \eta+D_{i} V D_{i} \eta-b_{i} V D_{i} \eta\right) d x d t \geq 4 \pi \int_{\Gamma \cap Q_{R}^{1, \frac{1}{4}}\left(0 ;-\frac{3}{4} R^{2}\right)} V \eta d x_{3} d t .
$$

We take $\eta$ such that

$\eta \equiv 1$ in $Q_{R}^{\frac{1}{2}, \frac{1}{8}}\left(0 ;-\frac{13}{16} R^{2}\right) ; \quad \eta \equiv 0$ outside $Q_{R}^{1, \frac{1}{4}}\left(0 ;-\frac{3}{4} R^{2}\right) ; \quad\left|\partial_{t} \eta\right|+|D \eta|^{2}+|\Delta \eta| \leq \frac{C}{R^{2}}$.

Then (73) and $\left.V\right|_{\Gamma \cap Q_{R}^{2,1}} \geq k$ imply

$$
\begin{aligned}
& \frac{\pi}{2} k R^{3} \leq \int_{Q_{R}^{1, \frac{1}{4}}\left(0 ;-\frac{3}{4} R^{2}\right)} V\left(\left|\partial_{t} \eta\right|+|\Delta \eta|+|\mathbf{b}| \cdot|D \eta|\right) d x d t
\end{aligned}
$$

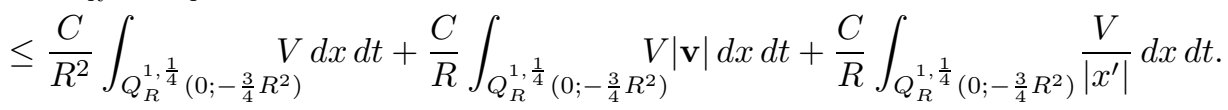

Splitting the integrals on the right-hand side into integrals over the set $\widehat{\mathcal{E}}_{\varkappa}$ and over its complement and recalling that $V \leq \mathfrak{N} k$ and $\frac{1}{\left|x^{\prime}\right|} \in L_{\frac{9}{5}, \infty}\left(Q_{R}^{1, \frac{1}{4}}\left(0 ;-\frac{3}{4} R^{2}\right)\right)$, we get

$$
\begin{aligned}
\frac{\pi}{2} k R^{3} \leq & \frac{C \mathfrak{N} k}{R^{2}}\left[\operatorname{meas}\left(\widehat{\mathcal{E}}_{\varkappa}\right)+R^{1+\alpha} \widehat{\mathcal{N}}\|\mathbf{1}\|_{q^{\prime}, \ell^{\prime}, \widehat{\mathcal{E}}_{\varkappa}}+R^{\frac{5}{3}}\|\mathbf{1}\|_{\frac{9}{4}, 1, \widehat{\mathcal{E}}_{\varkappa}}\right] \\
& +\frac{C \varkappa k}{R^{2}}\left[\operatorname{meas}\left(Q_{R}^{1, \frac{1}{4}}\right)+R^{1+\alpha} \widehat{\mathcal{N}}\|\mathbf{1}\|_{q^{\prime}, \ell^{\prime}, Q_{R}^{1, \frac{1}{4}}}+R^{\frac{5}{3}}\|\mathbf{1}\|_{\frac{9}{4}, 1, Q_{R}^{1, \frac{1}{4}}}\right] .
\end{aligned}
$$

The second term on the right-hand side is easily estimated by $C \varkappa k R^{3}\left(1+C_{17}(q, \ell) \widehat{\mathcal{N}}\right)$. Therefore, choosing $\varkappa=\varkappa(q, \ell, \widehat{\mathcal{N}})$ sufficiently small, we obtain

$$
\frac{C \mathfrak{N}}{R^{5}}\left[\operatorname{meas}\left(\widehat{\mathcal{E}}_{\varkappa}\right)+R^{1+\alpha} \widehat{\mathcal{N}}\|\mathbf{1}\|_{q^{\prime}, \ell^{\prime}, \widehat{\mathcal{E}}_{\varkappa}}+R^{\frac{5}{3}}\|\mathbf{1}\|_{\frac{9}{4}, 1, \widehat{\mathcal{E}}_{\varkappa}}\right] \geq 1 .
$$

To estimate the second term in brackets, we rewrite it as follows:

$$
\|\mathbf{1}\|_{q^{\prime}, \ell^{\prime}, \widehat{\mathcal{E}}_{\varkappa}}=\left(\int_{-R^{2}}^{-\frac{3}{4} R^{2}} \operatorname{meas}^{\frac{\ell^{\prime}}{q^{\prime}}}\left(\widehat{\mathcal{E}}_{\varkappa}(t)\right) d t\right)^{\frac{1}{\ell^{\prime}}} .
$$

If $q \leq \ell$, then, by the Hölder inequality,

$$
\|\mathbf{1}\|_{q^{\prime}, \ell^{\prime}, \widehat{\mathcal{E}}_{\varkappa}} \leq\left(\int_{-R^{2}}^{-\frac{3}{4} R^{2}} \operatorname{meas}\left(\widehat{\mathcal{E}}_{\varkappa}(t)\right) d t\right)^{\frac{1}{q^{\prime}}} \cdot\left(\frac{1}{4} R^{2}\right)^{\frac{1}{\ell^{\prime}}-\frac{1}{q^{\prime}}}=\operatorname{meas}^{\frac{1}{q^{\prime}}}\left(\widehat{\mathcal{E}}_{\varkappa}\right) \cdot\left(\frac{1}{4} R^{2}\right)^{\frac{1}{q}-\frac{1}{\ell}} .
$$

Otherwise, since meas $\left(\widehat{\mathcal{E}}_{\varkappa}(t)\right) \leq \operatorname{meas}\left(B_{R}\right)$, we obtain

$$
\begin{aligned}
\|\mathbf{1}\|_{q^{\prime}, \ell^{\prime}, \widehat{\mathcal{E}}_{\varkappa}} & =\left(\int_{-R^{2}}^{-\frac{3}{4} R^{2}}\left(\frac{\operatorname{meas}\left(\widehat{\mathcal{E}}_{\varkappa}(t)\right)}{\operatorname{meas}\left(B_{R}\right)}\right)^{\frac{\ell^{\prime}}{q^{\prime}}} d t\right)^{\frac{1}{\ell^{\prime}}} \cdot \operatorname{meas}^{\frac{1}{q^{\prime}}}\left(B_{R}\right) \\
& \leq\left(\int_{-R^{2}}^{-\frac{3}{4} R^{2}} \frac{\operatorname{meas}\left(\widehat{\mathcal{E}}_{\varkappa}(t)\right)}{\operatorname{meas}\left(B_{R}\right)} d t\right)^{\frac{1}{\ell^{\prime}}} \cdot \operatorname{meas}^{\frac{1}{q^{\prime}}}\left(B_{R}\right)=\operatorname{meas}^{\frac{1}{\ell^{\prime}}}\left(\widehat{\mathcal{E}}_{\varkappa}\right) \cdot\left(4 \pi R^{3}\right)^{\frac{1}{\ell}-\frac{1}{q}} .
\end{aligned}
$$

${ }^{7}$ In a particular case, this idea was used in CSTY. 
The third term in brackets in (174) is estimated similarly. Thus, we arrive at the following inequality for the quantity $\mathcal{A}=\frac{1}{R^{5}} \operatorname{meas}\left(\widehat{\mathcal{E}}_{\varkappa}\right)$ :

$$
\mathcal{A}+\mathcal{A}^{\frac{4}{9}}+\widehat{\mathcal{N}} \mathcal{A}^{1-\max \left\{\frac{1}{q}, \frac{1}{\ell}\right\}} \geq \frac{1}{C \mathfrak{N}}
$$

this yields (72). 1

Inequality (72) shows that

$$
\operatorname{meas}\left(\widehat{\mathcal{E}}_{\varkappa}(\bar{t})\right) \geq \delta \operatorname{meas}\left(B_{R}\right)
$$

for some $\bar{t} \in\left[-R^{2},-\frac{3}{4} R^{2}\right]$, and Corollary 3.3 ensures (71) with

$$
\widehat{\beta}_{3}=\varkappa \cdot \beta_{3}(\delta, 3,1, q, \ell, \widehat{\mathcal{N}}) .
$$

Theorem 4.3. Let $\mathbf{v}$ be an axisymmetric solution of the Navier-Stokes system in $\mathbb{R}^{3} \times$ $\mathbb{R}_{-}$. Also, suppose that $\mathbf{v}$ satisfies (59) with some $q$ and $\ell$ such that $\alpha \equiv \frac{3}{q}+\frac{2}{\ell}-1 \in[0,1[$. Let $u$ be a bounded Lipschitz solution of (67)-(68) with $\varepsilon=+1$ in $\mathbb{R}^{n} \times \mathbb{R}_{-}$. If $\left.u\right|_{\Gamma}=$ const, then $u \equiv$ const

Proof. Given $R$, we set $k=\frac{1}{2} \underset{Q_{2 R}}{\operatorname{osc}} u$ and consider two functions $V_{1}=u-\inf _{Q_{2 R}} u$ and $V_{2}=\sup _{Q_{2 R}} u-u$. At least one of them satisfies (70) with $\mathfrak{N}=2$. Thus, for this function Lemma 4.2 gives estimate (171), which implies (57) with

$$
\varkappa_{1}=1-\frac{1}{2} \widehat{\beta}_{3}(q, \ell, 2, \widehat{\mathcal{N}}) .
$$

Iteration of this inequality with respect to a suitable sequence $R_{m} \rightarrow \infty$ completes the proof.

\section{REFERENCES}

[DG] E. De Giorgi, Sulla differenziabilità e l'analiticità delle estremali degli integrali multipli regolari, Mem. Acad. Sci. Torino. Cl. Sci. Fis. Mat. Nat. (3) 3 (1957), 25-43. MR.0093649 (20:172)

[M] C. B. Morrey, Jr., Second order elliptic equations in several variables and Hölder continuity, Math. Z. 72 (1959/1960), 146-164. MR0120446(22:11200)

[Na] J. Nash, Parabolic equations, Proc. Nat. Acad. Sci. USA 43 (1957), 754-758. MR0089986 $(19: 749 \mathrm{~b})$

[LU1] O. A. Ladyzhenskaya and N. N. Ural'tseva, A boundary value-problem for linear and quasilinear parabolic equations, Dokl. Akad. Nauk SSSR 139 (1961), no. 3, 544-547; English transl. in Soviet Math. Dokl. 2 (1961). MR0141891 (25:5288)

[Mo1] J. Moser, On Harnack's theorem for elliptic differential equations, Comm. Pure Appl. Math. 14 (1961), 577-591. MR0159138(28:2356)

[Mo2] , A Harnack inequality for parabolic differential equations, Comm. Pure Appl. Math. 17 (1964), 101-134; Correction, Comm. Pure Appl. Math. 20 (1967), 231-236. MR0159139 (28:2357) MR0203268(34:3121)

[NU] A. I. Nazarov and N. N. Ural'tseva, Qualitative properties of solutions to elliptic and parabolic equations with unbounded lower-order coefficients, SPbMS El. Prepr. Archive. N 2009-05, 6 p.

[Z] Qi. S. Zhang, A strong regularity result for parabolic equations, Comm. Math. Phys. 244 (2004), no. 2, 245-260. MR.2031029(2005b:35116)

[KNSS] G. Koch, N. Nadirashvili, G. Seregin, and V. Šverák, Liouville theorems for the Navier-Stokes equations and applications, Acta Math. 203 (2009), no. 1, 83-105. MR2545826 (2010i:35281)

[CSTY] C.-C. Chen, R. M. Strain, T.-P. Tsai, and H.-T. Yau, Lower bound on the blow-up rate of the axisymmetric Navier-Stokes equations. I, Int. Math. Res. Not. 2008, no. 9, Art. ID rnn016, 31 pp.; II, Comm. Partial Differential Equations 34 (2009), no. 1-3, 203-232. MR2429247 (2009i:35233) MR 2512859 (2010f:35279)

[SSSZ] G. Seregin, L. Silvestre, V. Sverák, and A. Zlatos, On divergence-free drifts, Preprint arXiv:1010.6025v1.

[Tru] N. S. Trudinger, Linear elliptic operators with measurable coefficients, Ann. Scuola Norm. Sup. Pisa (3) 27 (1973), 265-308. MR0369884 (51:6113) 
[Li1] G. M. Lieberman, Second order parabolic differential equations, World Sci. Publ. Co., Inc., River Edge, NJ, 1996. MR1465184 (98k:35003)

[LU2] O. A. Ladyzhenskaya and N. N. Ural'tseva, Linear and quasilinear equations of elliptic type, 2nd ed., Nauka, Moscow, 1973; English transl. of 1st ed., Acad. Press, New York-London, 1968. MR0509265 (58:23009) MR0244627 (39:5941)

[LSU] O. A. Ladyzhenskaya, V. A. Solonnikov, and N. N. Ural'tseva, Linear and quasilinear equations of parabolic type, Nauka, Moscow, 1967; English transl., Transl. Math. Monogr., vol. 23, Amer. Math. Soc., Providence, RI, 1968. MR0241821(39:3159a) MR0241822 (39:3159b)

[LL] E. H. Lieb and M. Loss, Analysis, Grad. Stud. in Math., vol. 14, Amer. Math. Soc., Providence, RI, 1997. MR1415616 (98b:00004)

[S1] M. V. Safonov, Mean value theorems and Harnack inequalities for second-order parabolic equations, Nonlinear Problems in Mathematical Physics and Related Topics. II, Mezhdunar. Mat. Ser., vol. 2, Tamara Rozhkovskaya, Novosibirsk, 2002, pp. 299-320; English transl., Int. Math. Ser. (N.Y.), vol. 2, Kluwer/Plenum, New York, 2002, pp. 329-352. MR1972004 (2004d:35094)

[MV] V. G. Maz'ya and I. E. Verbitsky, Form boundedness of the general second order differential operator, Comm. Pure Appl. Math. 59 (2006), no. 9, 1286-1329. MR2237288 (2008d:47089)

[Tro] G. M. Troianiello, Elliptic differential equations and obstacle problems, Plenum Press, New York, 1987. MR:1094820(92b:35004)

[BIN] O. V. Besov, V. P. Il'in, and S. M. Nikol'skiǐ, Integral representations of functions and embedding theorems, 2nd ed., Nauka, Moscow, 1996; English transl. of 1st ed., Vol. I, II, V. H. Winston and Sons, Washington, DC, 1978, 1979. MR.1450401|(98b:46037) MR0519341|(80f:46030a) MR0521808 (80f:46030b)

[S2] M. V. Safonov, Non-divergence elliptic equations of second order with unbounded drift, Amer. Math. Soc. Transl. Ser. 2, vol. 229, Amer. Math. Soc., Providence, RI, 2010, pp. 211-232. MR2667641

Department of Mathematics and Mechanics, St. Petersburg State University, UniversitetSkaya Ul. 28, Stary Petergof, St. Petersburg 198504, Russia

E-mail address: al.il.nazarov@gmail.com

Department of Mathematics and Mechanics, St. Petersburg State University, UniversitetSkaya Ul. 28, Stary Petergof, St. Petersburg 198504, Russia

E-mail address: uunur@NU1253.spb.edu

Received 12/OCT/2010

Translated by THE AUTHORS 